\title{
Homological dimensions relative to preresolving subcategories
}

\author{
Zhaoyong Huang
}

\begin{abstract}
We introduce relative preresolving subcategories and precoresolving subcategories of an abelian category and define homological dimensions and codimensions relative to these subcategories, respectively. We study the properties of these homological dimensions and codimensions and unify some important properties possessed by some known homological dimensions. Then we apply the obtained properties to special subcategories and in particular to module categories. Finally we propose some open questions and conjectures which are closely related to the Auslander-Reiten conjecture and the strong Nakayama conjecture.
\end{abstract}

\section{Introduction}

In classical homological theory, homological dimensions are important and fundamental invariants, and every homological dimension of modules is defined relative to some certain subcategory of modules. For example, projective, flat, and injective dimensions of modules are defined relative to the categories of projective, flat, and injective modules, respectively. When projective, flat, and injective modules are generalized to Gorenstein projective, Gorenstein flat, and Gorenstein injective modules, respectively, in relative homological theory, Gorenstein projective, Gorenstein flat, and Gorenstein injective dimensions emerge; and, in particular, they share many nice properties of projective, flat, and injective dimensions, respectively (e.g., [AB], [C], [CFH], [CI], [DLM], [EJ1], [EJ2], [EJL], [GD], [GT], $[\mathrm{H} 1],[\mathrm{H} 2],[\mathrm{HuH}],[\mathrm{LHX}],[\mathrm{MD}],[\mathrm{SSW}],[\mathrm{Z}])$. Then a natural question is: if two homological (co)dimensions relative to a category and its subcategory are defined, what is the relation between these two homological (co)dimensions? The purpose of this paper is to study this question. We introduce relative preresolving subcategories and precoresolving subcategories and define homological dimensions and codimensions relative to these subcategories, respectively. Then we study

Kyoto Journal of Mathematics, Vol. 54, No. 4 (2014), 727-757

DOI $10.1215 / 21562261-2801795$, (C) 2014 by Kyoto University

Received March 4, 2013. Revised July 9, 2013. Accepted September 18, 2013.

2010 Mathematics Subject Classification: Primary 18G25; Secondary 18G20, 18G10, 16 E10.

Author's work partially supported by the Specialized Research Fund for the Doctoral Program of Higher Education (grant no. 20100091110034), Natural Science Foundation of China (grant no. 11171142), Natural Science Foundation of Jiangsu Province of China (grant no. BK2010007), and a project funded by the Priority Academic Program Development of Jiangsu Higher Education Institutions. 
their properties and unify some important properties possessed by some known homological dimensions.

This paper is organized as follows.

In Section 2, we give some terminology and some preliminary results; in particular, we give the definition of homological (co)dimension relative to a certain full and additive subcategory of an abelian category.

In Section 3, we first give the definition of (pre)resolving subcategories of an abelian category. Then we give some criteria for computing and comparing homological dimensions relative to different preresolving subcategories. Let $\mathscr{E}$ and $\mathscr{T}$ be additive and full subcategories of an abelian category $\mathscr{A}$ such that $\mathscr{T}$ is $\mathscr{E}$-preresolving with an $\mathscr{E}$-proper generator $\mathscr{C}$. Assume that $0 \rightarrow M \rightarrow T_{1} \rightarrow$ $T_{0} \rightarrow A \rightarrow 0$ is an exact sequence in $\mathscr{A}$ with both $T_{0}$ and $T_{1}$ objects in $\mathscr{T}$. Then there exists an exact sequence $0 \rightarrow M \rightarrow T \rightarrow C \rightarrow A \rightarrow 0$ in $\mathscr{A}$ with $T$ an object in $\mathscr{T}$ and $C$ an object in $\mathscr{C}$; and furthermore, if the former exact sequence is $\operatorname{Hom}_{\mathscr{A}}(X,-)$-exact for some object $X$ in $\mathscr{A}$, then so is the latter one. As applications of this result, we get that an object in $\mathscr{A}$ is an $n$ - $\mathscr{C}$-cosyzygy if and only if it is an $n$ - $\mathscr{T}$-cosyzygy; and we also get that the $\mathscr{T}$-dimension of an object $A$ in $\mathscr{A}$ is at most $n$ if and only if there exists an exact sequence $0 \rightarrow K_{n} \rightarrow C_{n-1} \rightarrow C_{n-2} \rightarrow \cdots \rightarrow C_{0} \rightarrow A \rightarrow 0$ in $\mathscr{A}$ with all $C_{i}$ 's objects in $\mathscr{C}$ and $K_{n}$ an object in $\mathscr{T}$. In addition, we give some sufficient conditions under which the $\mathscr{T}$-dimension and the $\mathscr{C}$-dimension of an object in $\mathscr{A}$ are identical.

Section 4 is completely dual to Section 3.

In Section 5, we apply the results in Sections 3 and 4 to special subcategories and in particular to module categories. Some known results are generalized. Finally we propose some questions and conjectures concerning the obtained results, which are closely related to the Auslander-Reiten conjecture and the strong Nakayama conjecture.

Throughout this paper, $\mathscr{A}$ is an abelian category and all subcategories of $\mathscr{A}$ are full and additive.

\section{Preliminaries}

In this section, we give some terminology and some preliminary results.

\section{DEFINITION 2.1 ([Hu, DEFINITION 5.2, P. 164])}

Let $\mathscr{C}$ be a subcategory of $\mathscr{A}$, and let $n \geq 0$.

(a) If there exists an exact sequence $0 \rightarrow M \rightarrow C_{n-1} \rightarrow C_{n-2} \rightarrow \cdots \rightarrow C_{0} \rightarrow$ $A \rightarrow 0$ in $\mathscr{A}$ with all $C_{i}$ 's objects in $\mathscr{C}$, then $M$ is called an $n$ - $\mathscr{C}$-syzygy object (of $A$ ), and $A$ is called an $n-\mathscr{C}$-cosyzygy object (of $M$ ); in this case, we denote $M=$ $\Omega_{\mathscr{C}}^{n}(A)$ and $A=\Omega_{\mathscr{C}}^{-n}(M)$. We denote by $\Omega_{\mathscr{C}}^{n}(\mathscr{A})$ (resp., $\Omega_{\mathscr{C}}^{-n}(\mathscr{A})$ ) the subcategory of $\mathscr{A}$ consisting of $n$ - $\mathscr{C}$-syzygy (resp., $n$ - $\mathscr{C}$-cosyzygy) objects.

(b) For an object $A$ in $\mathscr{A}$, the $\mathscr{C}$-dimension (resp., $\mathscr{C}$-codimension), denoted by $\mathscr{C}-\operatorname{dim} A$ (resp., $\mathscr{C}-\operatorname{codim} A)$, is defined as $\inf \{n \geq 0 \mid$ there exists an exact sequence $0 \rightarrow C_{n} \rightarrow \cdots \rightarrow C_{1} \rightarrow C_{0} \rightarrow A \rightarrow 0$ (resp., $0 \rightarrow A \rightarrow C^{0} \rightarrow C^{1} \rightarrow \cdots \rightarrow$ 
$C^{n} \rightarrow 0$ ) in $\mathscr{A}$ with all $C_{i}$ 's (resp., $C^{i}$ 's) objects in $\mathscr{C}$ \}. Set $\mathscr{C}-\operatorname{dim} A$ (resp., $\mathscr{C}-\operatorname{codim} A)=\infty$ if no such integer exists.

Let $\mathscr{C}$ be a subcategory of $\mathscr{A}$. We denote $\mathscr{C}^{\perp}=\left\{A\right.$ is an object in $\mathscr{A} \mid \operatorname{Ext}_{\mathscr{A}}^{i}(C$, $A)=0$ for any object $C$ in $\mathscr{C}$ and $i \geq 1\}$ and $\perp_{\mathscr{C}}=\{A$ is an object in $\mathscr{A} \mid$ $\operatorname{Ext}_{\mathscr{A}}^{i}(A, C)=0$ for any object $C$ in $\mathscr{C}$ and $\left.i \geq 1\right\}$.

\section{LEMMA 2.2}

Let $\mathscr{C}$ and $\mathscr{D}$ be subcategories of $\mathscr{A}$, let $M$ be an object in ${ }^{\perp} \mathscr{C}$, and let $M^{\prime}$ be an object in $\Omega_{\mathscr{C}}^{-n}(\mathscr{A})$ such that some $\Omega_{\mathscr{C}}^{n}\left(M^{\prime}\right)$ is an object in $\mathscr{D}^{\perp}$. If $\mathscr{D}-\operatorname{dim} M \leq$ $n(<\infty)$, then $\operatorname{Ext}_{\mathscr{A}}^{i}\left(M, M^{\prime}\right)=0$ for any $i \geq 1$.

Proof

By assumption, there exists an exact sequence

$$
0 \rightarrow M^{\prime \prime} \rightarrow C_{n-1} \rightarrow \cdots \rightarrow C_{1} \rightarrow C_{0} \rightarrow M^{\prime} \rightarrow 0
$$

in $\mathscr{A}$ with all $C_{i}$ 's objects in $\mathscr{C}$ and $M^{\prime \prime}$ an object in $\mathscr{D}^{\perp}$. Let $M$ be an object in $\perp_{\mathscr{C}}$. Then $\operatorname{Ext}_{\mathscr{A}}^{i}\left(M, M^{\prime}\right) \cong \operatorname{Ext}_{\mathscr{A}}^{n+i}\left(M, M^{\prime \prime}\right)$ for any $i \geq 1$. If $\mathscr{D}$-dim $M \leq$ $n(<\infty)$, then there exists an exact sequence

$$
0 \rightarrow D_{n} \rightarrow \cdots \rightarrow D_{1} \rightarrow D_{0} \rightarrow M \rightarrow 0
$$

in $\mathscr{A}$ with all $D_{i}$ 's objects in $\mathscr{D}$. So $\operatorname{Ext}_{\mathscr{A}}^{n+i}\left(M, M^{\prime \prime}\right) \cong \operatorname{Ext}_{\mathscr{A}}^{i}\left(D_{n}, M^{\prime \prime}\right)=0$ for any $i \geq 1$ and hence $\operatorname{Ext}_{\mathscr{A}}^{i}\left(M, M^{\prime}\right)=0$ for any $i \geq 1$.

Let $\mathscr{E}$ be a subcategory of $\mathscr{A}$. Recall from [EJ2] that a sequence

$$
\mathbb{S}: \cdots \rightarrow S_{1} \rightarrow S_{2} \rightarrow S_{3} \rightarrow \cdots
$$

in $\mathscr{A}$ is called $\operatorname{Hom}_{\mathscr{A}}(\mathscr{E},-)$-exact (resp., $\operatorname{Hom}_{\mathscr{A}}(-, \mathscr{E})$-exact) if $\operatorname{Hom}_{\mathscr{A}}(E, \mathbb{S})$ (resp., $\operatorname{Hom}_{\mathscr{A}}(\mathbb{S}, E)$ ) is exact for any object $E$ in $\mathscr{E}$. An epimorphism (resp., a monomorphism) in $\mathscr{A}$ is called $\mathscr{E}$-proper (resp., $\mathscr{E}$-coproper) if it is $\operatorname{Hom}_{\mathscr{A}}(\mathscr{E},-)$-exact (resp., $\operatorname{Hom}_{\mathscr{A}}(-, \mathscr{E})$-exact).

\section{PROPOSITION 2.3}

Let $\mathscr{C}$ and $\mathscr{E}$ be subcategories of $\mathscr{A}$, and let $\mathscr{C}$ be closed under kernels of $(\mathscr{E}-$ proper) epimorphisms. If

$$
0 \rightarrow A_{1} \rightarrow A_{2} \rightarrow A_{3} \rightarrow 0
$$

is a $\left(\operatorname{Hom}_{\mathscr{A}}(\mathscr{E},-)\right.$-exact) exact sequence in $\mathscr{A}$ with $A_{3}$ an object in $\mathscr{C}$, then $\mathscr{C}-\operatorname{dim} A_{1} \leq \mathscr{C}-\operatorname{dim} A_{2}$.

\section{Proof}

Let $\mathscr{C}-\operatorname{dim} A_{2}=n(<\infty)$, and let

$$
0 \rightarrow C_{n} \rightarrow \cdots \rightarrow C_{1} \rightarrow C_{0} \rightarrow A_{2} \rightarrow 0
$$

be an exact sequence in $\mathscr{A}$ with all $C_{i}$ 's objects in $\mathscr{C}$. By [Hu, Theorem 3.2], there exist exact sequences 


$$
0 \rightarrow C_{n} \rightarrow \cdots \rightarrow C_{1} \rightarrow C \rightarrow A_{1} \rightarrow 0
$$

and

$$
0 \rightarrow C \rightarrow C_{0} \rightarrow A_{3} \rightarrow 0 .
$$

From the proof of [Hu, Theorem 3.2] we see that if (2.1) is $\operatorname{Hom}_{\mathscr{A}}(\mathscr{E},-)$-exact, then so is (2.2). Because $\mathscr{C}$ is closed under kernels of ( $\mathscr{E}$-proper) epimorphisms and $A_{3}$ is an object in $\mathscr{C}$ by assumption, $C$ is an object in $\mathscr{C}$ and $\mathscr{C}-\operatorname{dim} A_{1} \leq n$.

Let $\mathscr{C}$ be a subcategory of $\mathscr{A}$. We write $\mathscr{C} \perp \mathscr{C}$ if $\operatorname{Ext}_{\mathscr{A}}^{i}\left(C_{1}, C_{2}\right)=0$ for any objects $C_{1}, C_{2}$ in $\mathscr{C}$ and $i \geq 1$, and we denote by $\mathscr{C}$-dim ${ }^{<\infty}$ (resp., $\mathscr{C}$-codim ${ }^{<\infty}$ ) the subcategory of $\mathscr{A}$ consisting of objects with finite $\mathscr{C}$-dimension (resp., $\mathscr{C}$-codimension).

\section{LEMMA 2.4}

Let $\mathscr{C}$ be a subcategory of $\mathscr{A}$ such that $\mathscr{C} \perp \mathscr{C}$ and $\mathscr{C}-\operatorname{dim}^{<\infty}$ is closed under direct summands, and let $0 \rightarrow K \rightarrow C \rightarrow A \rightarrow 0$ be an exact sequence in $\mathscr{A}$ with $\mathscr{C}-\operatorname{dim} A<\infty$ and $C$ an object in $\mathscr{C}$. If $K$ is an object in $\mathscr{C}^{\perp}$, then $\mathscr{C}-\operatorname{dim} K<\infty$.

Proof

Because $\mathscr{C}-\operatorname{dim} A<\infty$, there exists an exact sequence

$$
0 \rightarrow M \rightarrow C_{0} \rightarrow A \rightarrow 0
$$

in $\mathscr{A}$ with $C_{0}$ an object in $\mathscr{C}$ and $\mathscr{C}-\operatorname{dim} M<\infty$. Consider the following pullback diagram:

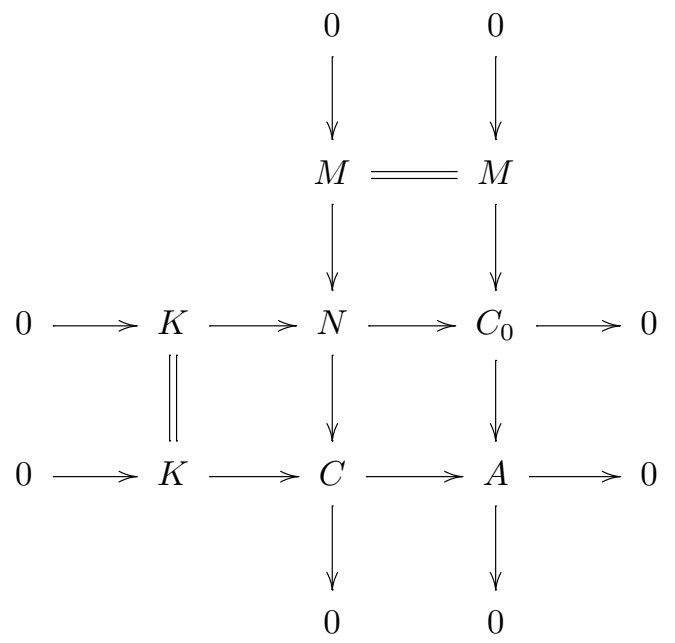

Because $\mathscr{C} \perp \mathscr{C}$ and $\mathscr{C}-\operatorname{dim} M<\infty$, it is easy to get that $M \in \mathscr{C}^{\perp}$ by dimension shifting. So the middle column in the above diagram splits, and hence $\mathscr{C}-\operatorname{dim} N \leq$ $\mathscr{C}-\operatorname{dim} M<\infty$ by [Hu, Lemma 3.1]. Because $K$ is an object in $\mathscr{C}^{\perp}$ by assumption, 
the middle row in the above diagram also splits and $K$ is isomorphic to a direct summand of $N$. Thus $\mathscr{C}-\operatorname{dim} K<\infty$.

\section{DEFINITION 2.5}

Let $\mathscr{C} \subseteq \mathscr{T}$ be subcategories of $\mathscr{A}$.

(a) $\mathscr{C}$ is called a generator (resp., cogenerator) for $\mathscr{T}$ if, for any object $T$ in $\mathscr{T}$, there exists an exact sequence $0 \rightarrow T^{\prime} \rightarrow C \rightarrow T \rightarrow 0$ (resp., $0 \rightarrow T \rightarrow C \rightarrow$ $\left.T^{\prime} \rightarrow 0\right)$ in $\mathscr{T}$ with $C$ an object in $\mathscr{C}$ (cf. [SSW]).

(b) Let $\mathscr{E}$ be a subcategory of $\mathscr{A}$. Then, $\mathscr{C}$ is called an $\mathscr{E}$-proper generator (resp., $\mathscr{E}$-coproper cogenerator) for $\mathscr{T}$ if, for any object $T$ in $\mathscr{T}$, there exists a $\operatorname{Hom}_{\mathscr{A}}(\mathscr{E},-)\left(\right.$ resp., $\left.\operatorname{Hom}_{\mathscr{A}}(-, \mathscr{E})\right)$-exact exact sequence $0 \rightarrow T^{\prime} \rightarrow C \rightarrow T \rightarrow 0$ (resp., $0 \rightarrow T \rightarrow C \rightarrow T^{\prime} \rightarrow 0$ ) in $\mathscr{A}$ such that $C$ is an object in $\mathscr{C}$ and $T^{\prime}$ is an object in $\mathscr{T}$.

\section{LEMMA 2.6}

Let $\mathscr{C} \subseteq \mathscr{T}$ be subcategories of $\mathscr{A}$ such that $\mathscr{C}$ is a cogenerator for $\mathscr{T}$, and let $0 \rightarrow A_{1} \rightarrow A_{2} \rightarrow A_{3} \rightarrow 0$ be an exact sequence in $\mathscr{A}$ such that both $A_{2}$ and $A_{3}$ are objects in $\mathscr{T}^{\perp}$. If $A_{1}$ is an object in $\mathscr{C}^{\perp}$, then $A_{1}$ is an object in $\mathscr{T}^{\perp}$.

\section{Proof}

Let $0 \rightarrow A_{1} \rightarrow A_{2} \rightarrow A_{3} \rightarrow 0$ be an exact sequence in $\mathscr{A}$ such that both $A_{2}$ and $A_{3}$ are objects in $\mathscr{T}^{\perp}$. Then $\operatorname{Ext}_{\mathscr{A}}^{i}\left(T, A_{1}\right)=0$ for any object $T$ in $\mathscr{T}$ and $i \geq 2$. Because $\mathscr{C}$ is a cogenerator for $\mathscr{T}$ by assumption, there exists an exact sequence

$$
0 \rightarrow T \rightarrow C \rightarrow T^{\prime} \rightarrow 0
$$

in $\mathscr{A}$ with $C$ an object in $\mathscr{C}$ and $T^{\prime}$ an object in $\mathscr{T}$, which yields an exact sequence

$$
\operatorname{Ext}_{\mathscr{A}}^{i}\left(C, A_{1}\right) \rightarrow \operatorname{Ext}_{\mathscr{A}}^{i}\left(T, A_{1}\right) \rightarrow \operatorname{Ext}_{\mathscr{A}}^{i+1}\left(T^{\prime}, A_{1}\right)
$$

for any $i \geq 1$. Note that $\operatorname{Ext}_{\mathscr{A}}^{i+1}\left(T^{\prime}, A_{1}\right)=0$ for any $i \geq 1$ by the above argument. So, if $A_{1}$ is an object in $\mathscr{C}^{\perp}$, then $\operatorname{Ext}_{\mathscr{A}}^{i}\left(T, A_{1}\right)=0$ for any $i \geq 1$ and $A_{1}$ is an object in $\mathscr{T}^{\perp}$.

\section{LEMMA 2.7}

Let $\mathscr{C} \subseteq \mathscr{T}$ be subcategories of $\mathscr{A}$ such that $\mathscr{C}$ is a cogenerator for $\mathscr{T}$ and $\mathscr{C}$ is closed under direct summands. Then $\mathscr{T} \cap \mathscr{T}^{\perp} \subseteq \mathscr{C}$.

\section{Proof}

Let $T$ be an object in $\mathscr{T} \cap \mathscr{T}^{\perp}$. Then there exists a split exact sequence

$$
0 \rightarrow T \rightarrow C \rightarrow T^{\prime} \rightarrow 0
$$

in $\mathscr{A}$ with $C$ an object in $\mathscr{C}$ and $T^{\prime}$ an object in $\mathscr{T}$. So $T$ is isomorphic to a direct summand of $C$. Because $\mathscr{C}$ is closed under direct summands by assumption, $T$ is an object in $\mathscr{C}$. 
Sather-Wagstaff, Sharif, and White introduced the Gorenstein category $\mathcal{G}(\mathscr{C})$ as follows.

DEFINITION 2.8 ([SSW, DEFINITION 4.1])

Let $\mathscr{C}$ be a subcategory of $\mathscr{A}$. The Gorenstein subcategory $\mathcal{G}(\mathscr{C})$ of $\mathscr{A}$ is defined as $\mathcal{G}(\mathscr{C})=\left\{A\right.$ is an object in $\mathscr{A} \mid$ there exists an exact sequence $\cdots \rightarrow C_{1} \rightarrow C_{0} \rightarrow$ $C^{0} \rightarrow C^{1} \rightarrow \cdots$ in $\mathscr{A}$ with all terms objects in $\mathscr{C}$, which is both $\operatorname{Hom}_{\mathscr{A}}(\mathscr{C},-)$ exact and $\operatorname{Hom}_{\mathscr{A}}(-, \mathscr{C})$-exact such that $\left.A \cong \operatorname{Im}\left(C_{0} \rightarrow C^{0}\right)\right\}$.

The Gorenstein category unifies the following notions: modules of Gorenstein dimension zero (see $[\mathrm{AB}]$ ), Gorenstein projective modules, Gorenstein injective modules (see [EJ1]), $V$-Gorenstein projective modules, $V$-Gorenstein injective modules (see [EJL]), $\mathcal{W}$-Gorenstein modules (see [GD]), and so on (see $[\mathrm{Hu}]$ for the details).

\section{Computation and comparison of homological dimensions}

In this section, we introduce the notion of (pre)resolving subcategories of $\mathscr{A}$. Then we give some criteria for computing and comparing homological dimensions relative to different preresolving subcategories.

\section{DEFINITION 3.1}

Let $\mathscr{E}$ and $\mathscr{T}$ be subcategories of $\mathscr{A}$. Then $\mathscr{T}$ is called $\mathscr{E}$-preresolving in $\mathscr{A}$ if the following conditions are satisfied.

(a) $\mathscr{T}$ admits an $\mathscr{E}$-proper generator.

(b) $\mathscr{T}$ is closed under $\mathscr{E}$-proper extensions, that is, for any $\operatorname{Hom}_{\mathscr{A}}(\mathscr{E},-)$ exact exact sequence $0 \rightarrow A_{1} \rightarrow A_{2} \rightarrow A_{3} \rightarrow 0$ in $\mathscr{A}$, if both $A_{1}$ and $A_{3}$ are objects in $\mathscr{T}$, then $A_{2}$ is also an object in $\mathscr{T}$.

An $\mathscr{E}$-preresolving subcategory $\mathscr{T}$ of $\mathscr{A}$ is called $\mathscr{E}$-resolving if the following condition is satisfied.

(c) $\mathscr{T}$ is closed under kernels of $\mathscr{E}$-proper epimorphisms, that is, for any $\operatorname{Hom}_{\mathscr{A}}(\mathscr{E},-)$-exact exact sequence $0 \rightarrow A_{1} \rightarrow A_{2} \rightarrow A_{3} \rightarrow 0$ in $\mathscr{A}$, if both $A_{2}$ and $A_{3}$ are objects in $\mathscr{T}$, then $A_{1}$ is also an object in $\mathscr{T}$.

The following list shows that the class of $\mathscr{E}$-(pre)resolving subcategories is rather large.

EXAMPLE $3.2 \quad$ (a) Let $\mathscr{A}$ admit enough projective objects, and let $\mathscr{E}$ be the subcategory of $\mathscr{A}$ consisting of projective objects. Then a subcategory of $\mathscr{A}$ closed under $\mathscr{E}$-proper extensions is just a subcategory of $\mathscr{A}$ closed under extensions. Furthermore, if $\mathscr{C}=\mathscr{E}$ in the above definition, then an $\mathscr{E}$-preresolving subcategory is just a subcategory which contains all projective objects and is closed 
under extensions, and an $\mathscr{E}$-resolving subcategory is just a projectively resolving subcategory in the sense of [H1].

(b) Let $\mathscr{C}$ be a subcategory of $\mathscr{A}$ with $\mathscr{C} \perp \mathscr{C}$. Then by [SSW, Corollary 4.5], the Gorenstein subcategory $\mathcal{G}(\mathscr{C})$ of $\mathscr{A}$ is a $\mathscr{C}$-preresolving subcategory of $\mathscr{A}$ with a $\mathscr{C}$-proper generator $\mathscr{C}$; furthermore, if $\mathscr{C}$ is closed under kernels of epimorphisms, then $\mathcal{G}(\mathscr{C})$ is a $\mathscr{C}$-resolving subcategory of $\mathscr{A}$ by [SSW, Theorem 4.12(a)].

(c) Let $R$ be a ring, let $\operatorname{Mod} R$ be the category of left $R$-modules, and let $\mathcal{P}(\operatorname{Mod} R)$ be the subcategory of $\operatorname{Mod} R$ consisting of projective modules. Recall from [EJ2] that a pair of subcategories $(\mathscr{X}, \mathscr{Y})$ of $\operatorname{Mod} R$ is called a cotorsion pair if $\mathscr{X}=\left\{X \in \operatorname{Mod} R \mid \operatorname{Ext}_{R}^{1}(X, Y)=0\right.$ for any $\left.Y \in \mathscr{Y}\right\}$ and $\mathscr{Y}=\{Y \in \operatorname{Mod} R \mid$ $\operatorname{Ext}_{R}^{1}(X, Y)=0$ for any $\left.X \in \mathscr{X}\right\}$. If $(\mathscr{X}, \mathscr{Y})$ is a cotorsion pair in $\operatorname{Mod} R$, then $\mathscr{X}$ is a $\mathcal{P}(\operatorname{Mod} R)$-preresolving subcategory of $\operatorname{Mod} R$ with a $\mathcal{P}(\operatorname{Mod} R)$-proper generator $\mathcal{P}(\operatorname{Mod} R)$ (see [EJ2]).

(d) Let $R$ be a ring, and let $\mathcal{F}(\operatorname{Mod} R)$ be the subcategory of $\operatorname{Mod} R$ consisting of flat modules. Then by [Hu, Lemma 3.1 and Theorem 3.2], it is not difficult to see that the subcategory of $\operatorname{Mod} R$ consisting of strongly Gorenstein flat modules (see [DLM] or Section 5 below for the definition) is an $\mathcal{F}(\operatorname{Mod} R)$-resolving subcategory of $\operatorname{Mod} R$ with an $\mathcal{F}(\operatorname{Mod} R)$-proper generator $\mathcal{P}(\operatorname{Mod} R)$.

(e) Let $R$ be a ring. Then, the subcategory of $\operatorname{Mod} R$ consisting of the modules $A$ satisfying $\operatorname{Ext}_{R}^{i}(A, P)=0$ for any $P \in \mathcal{P}(\operatorname{Mod} R)$ and $i \geq 1$, is a $\mathcal{P}(\operatorname{Mod} R)$ resolving subcategory of $\operatorname{Mod} R$ with a $\mathcal{P}(\operatorname{Mod} R)$-proper generator $\mathcal{P}(\operatorname{Mod} R)$. Let $R$ be a left Noetherian $\operatorname{ring}$, let $\bmod R$ be the category of finitely generated left $R$-modules, and let $\mathcal{P}(\bmod R)$ be the subcategory of $\bmod R$ consisting of projective modules. Then the subcategory of $\bmod R$ consisting of the modules $A$ satisfying $\operatorname{Ext}_{R}^{i}(A, R)=0$ for any $i \geq 1$ is a $\mathcal{P}(\bmod R)$-resolving subcategory of $\bmod R$ with a $\mathcal{P}(\bmod R)$-proper generator $\mathcal{P}(\bmod R)$.

Unless stated otherwise, in the rest of this section, we fix a subcategory $\mathscr{E}$ of $\mathscr{A}$ and an $\mathscr{E}$-preresolving subcategory $\mathscr{T}$ of $\mathscr{A}$ admitting an $\mathscr{E}$-proper generator $\mathscr{C}$. We will give some criteria for computing the $\mathscr{T}$-dimension of a given object $A$ in $\mathscr{A}$, and then compare it with the $\mathscr{C}$-dimension of $A$.

The following two propositions play a crucial role in this section.

\section{PROPOSITION 3.3}

Let

$$
0 \rightarrow M \rightarrow T_{1} \stackrel{f}{\longrightarrow} T_{0} \rightarrow A \rightarrow 0
$$

be an exact sequence in $\mathscr{A}$ with both $T_{0}$ and $T_{1}$ objects in $\mathscr{T}$. Then we have the following.

(a) There exists an exact sequence

$$
0 \rightarrow M \rightarrow T \rightarrow C \rightarrow A \rightarrow 0
$$

in $\mathscr{A}$ with $T$ an object in $\mathscr{T}$ and $C$ an object in $\mathscr{C}$.

(b) If (3.1) is $\operatorname{Hom}_{\mathscr{A}}(X,-)$-exact for some object $X$ in $\mathscr{A}$, then so is (3.2). 
Proof

(a) Let

$$
0 \rightarrow M \rightarrow T_{1} \stackrel{f}{\longrightarrow} T_{0} \rightarrow A \rightarrow 0
$$

be an exact sequence in $\mathscr{A}$ with both $T_{0}$ and $T_{1}$ objects in $\mathscr{T}$. Because there exists a $\operatorname{Hom}_{\mathscr{A}}(\mathscr{E},-)$-exact exact sequence

$$
0 \rightarrow T_{0}^{\prime} \rightarrow C \rightarrow T_{0} \rightarrow 0
$$

in $\mathscr{A}$ with $C$ an object in $\mathscr{C}$ and $T_{0}^{\prime}$ an object in $\mathscr{T}$, we have the following pullback diagram:

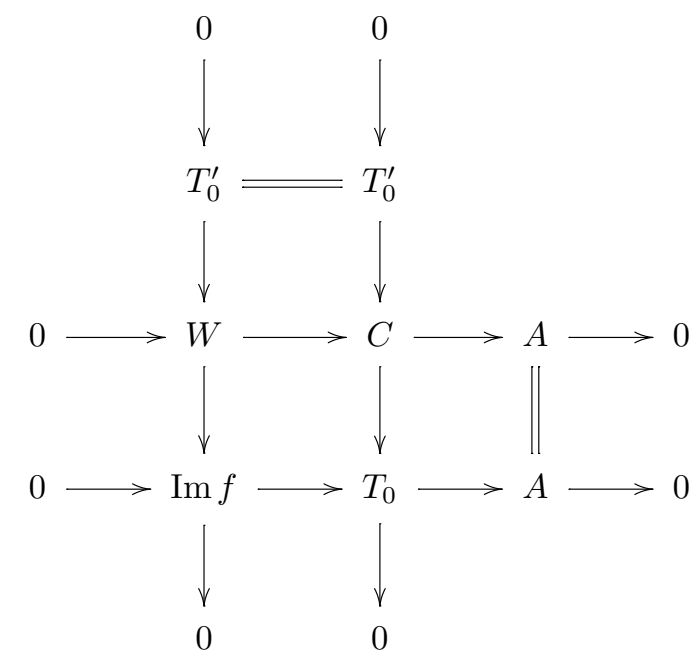

Then consider the following pullback diagram:

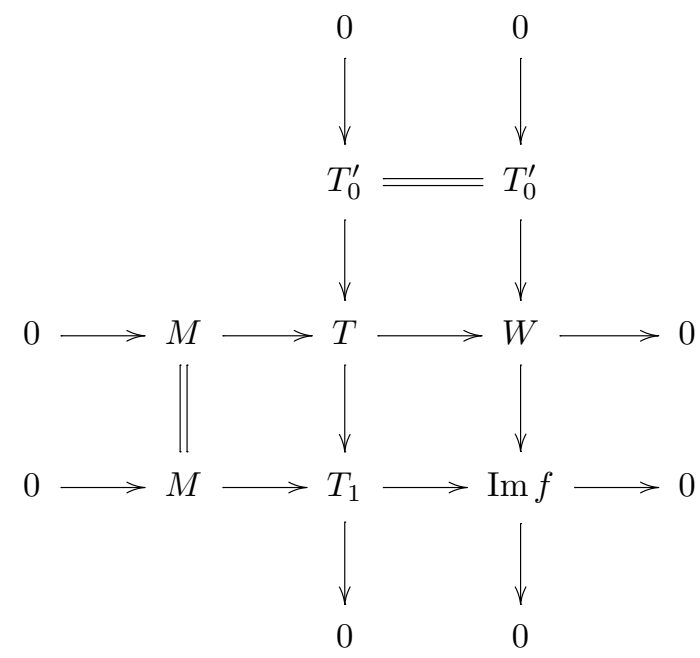

Because the middle column in the first diagram is $\operatorname{Hom}_{\mathscr{A}}(\mathscr{E},-)$-exact, the first column in the first diagram (that is, the third column in the second diagram) and the middle column in the second diagram are also $\operatorname{Hom}_{\mathscr{A}}(\mathscr{E},-)$-exact by $[\mathrm{Hu}$, 
Lemma 2.4(1)]. Because both $T_{0}^{\prime}$ and $T_{1}$ are objects in $\mathscr{T}, T$ is also an object in $\mathscr{T}$. Connecting the middle rows in the above two diagrams we get the desired exact sequence.

(b) If (3.1) is $\operatorname{Hom}_{\mathscr{A}}(X,-)$-exact for some object $X$ in $\mathscr{A}$, then so are the third rows in the above two diagrams. So the middle rows in the above two diagrams and (3.2) are also $\operatorname{Hom}_{\mathscr{A}}(X,-)$-exact by [Hu, Lemma 2.4(1)].

As an application of Proposition 3.3, we get the following.

\section{PROPOSITION 3.4}

Let $n \geq 1$, and let

$$
0 \rightarrow M \rightarrow T_{n-1} \rightarrow T_{n-2} \rightarrow \cdots \rightarrow T_{0} \rightarrow A \rightarrow 0
$$

be an exact sequence in $\mathscr{A}$ with all $T_{i}$ 's objects in $\mathscr{T}$. Then there exist an exact sequence

$$
0 \rightarrow N \rightarrow C_{n-1} \rightarrow C_{n-2} \rightarrow \cdots \rightarrow C_{0} \rightarrow A \rightarrow 0
$$

and a $\operatorname{Hom}_{\mathscr{A}}(\mathscr{E},-)$-exact exact sequence

$$
0 \rightarrow T \rightarrow N \rightarrow M \rightarrow 0
$$

in $\mathscr{A}$ with all $C_{i}$ 's objects in $\mathscr{C}$ and $T$ an object in $\mathscr{T}$. In particular, an object in $\mathscr{A}$ is an $n-\mathscr{C}$-cosyzygy if and only if it is an $n-\mathscr{T}$-cosyzygy.

\section{Proof}

We proceed by induction on $n$. The case for $n=1$ has been proved in the proof of Proposition 3.3. Now suppose that $n \geq 2$, and suppose that we have an exact sequence

$$
0 \rightarrow M \rightarrow T_{n-1} \rightarrow T_{n-2} \rightarrow \cdots \rightarrow T_{0} \rightarrow A \rightarrow 0
$$

in $\mathscr{A}$ with all $T_{i}$ 's objects in $\mathscr{T}$. Put $K=\operatorname{Ker}\left(T_{1} \rightarrow T_{0}\right)$. By Proposition 3.3, we get an exact sequence

$$
0 \rightarrow K \rightarrow T_{1}^{\prime} \rightarrow C_{0} \rightarrow A \rightarrow 0
$$

in $\mathscr{A}$ with $T_{1}^{\prime}$ an object in $\mathscr{T}$ and $C_{0}$ an object in $\mathscr{C}$. Put $A^{\prime}=\operatorname{Im}\left(T_{1}^{\prime} \rightarrow C_{0}\right)$. Then we get an exact sequence

$$
0 \rightarrow M \rightarrow T_{n-1} \rightarrow T_{n-2} \rightarrow \cdots \rightarrow T_{2} \rightarrow T_{1}^{\prime} \rightarrow A^{\prime} \rightarrow 0
$$

in $\mathscr{A}$. Thus the assertion follows from the induction hypothesis.

The following corollary is an immediate consequence of Proposition 3.4.

\section{COROLLARY 3.5}

Let $M$ be an object in $\mathscr{A}$, and let $n \geq 0$. If $\mathscr{T}$-codim $M=n$, then there exists a $\operatorname{Hom}_{\mathscr{A}}(\mathscr{E},-)$-exact exact sequence $0 \rightarrow T \rightarrow N \rightarrow M \rightarrow 0$ in $\mathscr{A}$ with $\mathscr{C}-\operatorname{codim} N \leq$ $n$ and $T$ an object in $\mathscr{T}$. 
Proof

Let $M$ be an object in $\mathscr{A}$ with $\mathscr{T}$-codim $M=n$. Applying Proposition 3.4 with $A=0$ we get a $\operatorname{Hom}_{\mathscr{A}}(\mathscr{E},-)$-exact exact sequence $0 \rightarrow T \rightarrow N \rightarrow M \rightarrow 0$ in $\mathscr{A}$ with $\mathscr{C}-\operatorname{codim} N \leq n$ and $T$ an object in $\mathscr{T}$.

We give a criterion for computing the $\mathscr{T}$-dimension of an object in $\mathscr{A}$ as follows.

\section{THEOREM 3.6}

The following statements are equivalent for any object $A$ in $\mathscr{A}$ and $n \geq 0$.

(a) $\mathscr{T}-\operatorname{dim} A \leq n$.

(b) There exists an exact sequence

$$
0 \rightarrow K_{n} \rightarrow C_{n-1} \rightarrow C_{n-2} \rightarrow \cdots \rightarrow C_{0} \rightarrow A \rightarrow 0
$$

in $\mathscr{A}$ with all $C_{i}$ 's objects in $\mathscr{C}$ and $K_{n}$ an object in $\mathscr{T}$.

Proof

The proof of $(b) \Rightarrow(a)$ is trivial.

For $(\mathrm{a}) \Rightarrow(\mathrm{b})$, we proceed by induction on $n$. The case for $n=0$ is trivial. If $n=1$, then there exists an exact sequence

$$
0 \rightarrow T_{1} \rightarrow T_{0} \rightarrow A \rightarrow 0
$$

in $\mathscr{A}$ with both $T_{0}$ and $T_{1}$ objects in $\mathscr{T}$. Applying Proposition 3.3 with $M=0$, we get an exact sequence

$$
0 \rightarrow K_{1} \rightarrow C_{0} \rightarrow A \rightarrow 0
$$

in $\mathscr{A}$ with $C_{0}$ an object in $\mathscr{C}$ and $K_{1}$ an object in $\mathscr{T}$.

Now suppose that $n \geq 2$. Then there exists an exact sequence

$$
0 \rightarrow T_{n} \rightarrow T_{n-1} \rightarrow \cdots \rightarrow T_{1} \rightarrow T_{0} \rightarrow A \rightarrow 0
$$

in $\mathscr{A}$ with all $T_{i}$ 's objects in $\mathscr{T}$. Put $M=\operatorname{Ker}\left(T_{1} \rightarrow T_{0}\right)$. By Proposition 3.3, we get an exact sequence

$$
0 \rightarrow M \rightarrow T_{1}^{\prime} \rightarrow C_{0} \rightarrow A \rightarrow 0
$$

in $\mathscr{A}$ with $C_{0}$ an object in $\mathscr{C}$ and $T_{1}^{\prime}$ an object in $\mathscr{T}$. Put $B=\operatorname{Im}\left(T_{1}^{\prime} \rightarrow C_{0}\right)$. Then we get an exact sequence

$$
0 \rightarrow T_{n} \rightarrow T_{n-1} \rightarrow \cdots \rightarrow T_{1}^{\prime} \rightarrow B \rightarrow 0
$$

By the induction hypothesis, we get the exact sequence

$$
0 \rightarrow K_{n} \rightarrow C_{n-1} \rightarrow \cdots \rightarrow C_{1} \rightarrow B \rightarrow 0
$$

in $\mathscr{A}$ with all $C_{i}$ 's objects in $\mathscr{C}$ and $K_{n}$ an object in $\mathscr{T}$. Thus we get the desired exact sequence

$$
0 \rightarrow K_{n} \rightarrow C_{n-1} \rightarrow C_{n-2} \rightarrow \cdots \rightarrow C_{1} \rightarrow C_{0} \rightarrow A \rightarrow 0 .
$$


The following result gives a criterion for computing the $\mathscr{T}$-codimension of an object in $\mathscr{A}$. To some extent, the proof of this result is dual to that of Theorem 3.6, so we omit it.

\section{THEOREM 3.7}

The following statements are equivalent for any object $M$ in $\mathscr{A}$ and $n \geq 0$.

(a) $\mathscr{T}-\operatorname{codim} M \leq n$.

(b) There exists an exact sequence

$$
0 \rightarrow M \rightarrow K^{0} \rightarrow C^{1} \rightarrow \cdots \rightarrow C^{n-1} \rightarrow C^{n} \rightarrow 0
$$

in $\mathscr{A}$ with $K^{0}$ an object in $\mathscr{T}$ and all $C^{i}$ 's objects in $\mathscr{C}$, that is, there exists an exact sequence

$$
0 \rightarrow M \rightarrow T \rightarrow H \rightarrow 0
$$

in $\mathscr{A}$ with $T$ an object in $\mathscr{T}$ and $\mathscr{C}-\operatorname{codim} H \leq n-1$.

The following result gives a sufficient condition such that the $n$ - $\mathscr{C}$-syzygy of an object in $\mathscr{A}$ with $\mathscr{T}$-dimension at most $n$ is in $\mathscr{T}$, in which the first assertion generalizes [AB, Lemma 3.12].

\section{THEOREM 3.8}

Let $\mathscr{T}$ be closed under kernels of (E्E-proper) epimorphisms and $\mathscr{T} \subseteq \mathscr{C}^{\perp}$, and let $n \geq 0$. Then for any object $A$ in $\mathscr{A}$ with $\mathscr{T}-\operatorname{dim} A \leq n$ we have the following.

(a) For any $\left(\operatorname{Hom}_{\mathscr{A}}(\mathscr{E},-)\right.$-exact) exact sequence $0 \rightarrow K_{n} \rightarrow C_{n-1} \rightarrow \cdots \rightarrow$ $C_{1} \rightarrow C_{0} \rightarrow A \rightarrow 0$ in $\mathscr{A}$ with all $C_{i}$ 's objects in $\mathscr{C}, K_{n}$ is an object in $\mathscr{T}$.

(b) If $0 \rightarrow K \rightarrow C \rightarrow A \rightarrow 0$ is a ( $\operatorname{Hom}_{\mathscr{A}}(\mathscr{E},-)$-exact) exact sequence in $\mathscr{A}$ with $C$ an object in $\mathscr{C}$, then $\mathscr{T}-\operatorname{dim} K \leq n-1$.

Proof

Let $\mathscr{T}-\operatorname{dim} A \leq n$, and let $\mathscr{T} \subseteq \mathscr{C}^{\perp}$. Then there exists a $\operatorname{Hom}_{\mathscr{A}}(\mathscr{C},-)$-exact exact sequence

$$
0 \rightarrow T_{n} \rightarrow C_{n-1}^{\prime} \rightarrow \cdots \rightarrow C_{1}^{\prime} \rightarrow C_{0}^{\prime} \rightarrow A \rightarrow 0
$$

in $\mathscr{A}$ with all $C_{i}^{\prime}$ 's objects in $\mathscr{C}$ and $T_{n}$ an object in $\mathscr{T}$ by Proposition 3.4.

(a) Let

$$
0 \rightarrow K_{n} \rightarrow C_{n-1} \rightarrow \cdots \rightarrow C_{1} \rightarrow C_{0} \rightarrow A \rightarrow 0
$$

be a $\left(\operatorname{Hom}_{\mathscr{A}}(\mathscr{E},-)\right.$-exact) exact sequence in $\mathscr{A}$ with all $C_{i}$ 's objects in $\mathscr{C}$. Then by $[\mathrm{Hu}$, Theorem 3.2$]$ we get a $\left(\operatorname{Hom}_{\mathscr{A}}(\mathscr{E},-)\right.$-exact $)$ exact sequence

$$
0 \rightarrow K_{n} \rightarrow T_{n} \oplus C_{n-1} \rightarrow C_{n-1}^{\prime} \oplus C_{n-2} \rightarrow \cdots \rightarrow C_{1}^{\prime} \oplus C_{0} \rightarrow C_{0}^{\prime} \rightarrow 0 .
$$

Because $\mathscr{T}$ is closed under kernels of ( $\mathscr{E}$-proper) epimorphisms by assumption, $K_{n}$ is an object in $\mathscr{T}$. 
(b) Put $T_{1}=\operatorname{Im}\left(C_{1}^{\prime} \rightarrow C_{0}^{\prime}\right)$. Then we have a $\operatorname{Hom}_{\mathscr{A}}(\mathscr{C},-)$-exact exact sequence

$$
0 \rightarrow T_{1} \rightarrow C_{0}^{\prime} \rightarrow A \rightarrow 0
$$

in $\mathscr{A}$ with $\mathscr{T}-\operatorname{dim} T_{1} \leq n-1$. Let $0 \rightarrow K \rightarrow C \rightarrow A \rightarrow 0$ be a $\left(\operatorname{Hom}_{\mathscr{A}}(\mathscr{E},-)\right.$-exact $)$ exact sequence in $\mathscr{A}$ with $C$ an object in $\mathscr{C}$. Consider the following pullback diagram:

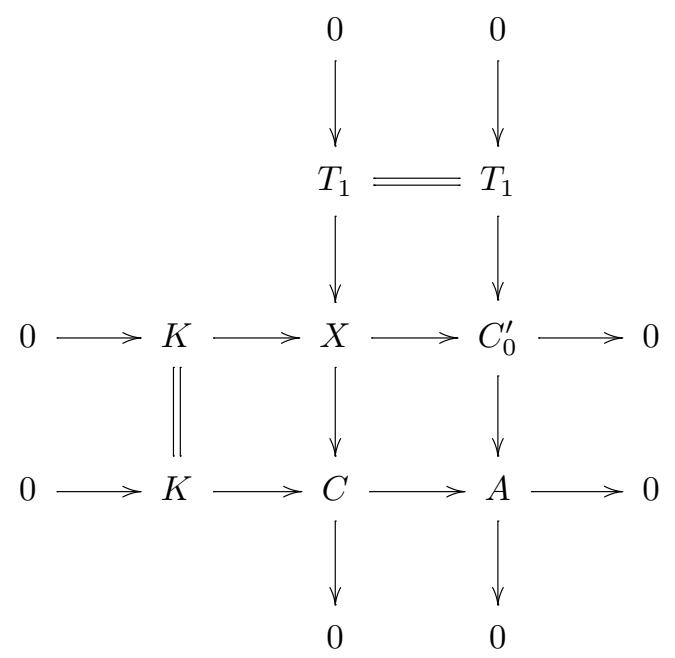

Because the third column in this diagram is $\operatorname{Hom}_{\mathscr{A}}(\mathscr{C},-)$-exact, the middle column is also $\operatorname{Hom}_{\mathscr{A}}(\mathscr{C},-)$-exact by $[\mathrm{Hu}$, Lemma 2.4(1)]. So the middle column splits and $X \cong T_{1} \oplus C$. Then the middle row yields an exact sequence

$$
0 \rightarrow K \rightarrow T_{1} \oplus C \rightarrow C_{0}^{\prime} \rightarrow 0 \text {. }
$$

By Proposition 2.3, $\mathscr{T}-\operatorname{dim} K \leq \mathscr{T}-\operatorname{dim} T_{1} \oplus C \leq n-1$.

We use $\mathscr{T}$-dim ${ }^{\leq n}$ to denote the subcategory of $\mathscr{A}$ consisting of objects with $\mathscr{T}$-dimension at most $n$.

\section{COROLLARY 3.9}

Let $\mathscr{T}$ be a $\mathscr{C}$-resolving subcategory of $\mathscr{A}$ with a $\mathscr{C}$-proper generator $\mathscr{C}$ and $\mathscr{T} \subseteq \mathscr{C}^{\perp}$. If $\mathscr{T}$ is closed under direct summands, then so is $\mathscr{T}$-dim ${ }^{\leq n}$ for any $n \geq 0$.

\section{Proof}

The case for $n=0$ follows from assumption. Now, let $n \geq 1$, and let $A$ be an object in $\mathscr{A}$ with $\mathscr{T}-\operatorname{dim} A \leq n$ and $A=A_{1} \oplus A_{2}$. Because $\mathscr{T} \subseteq \mathscr{C}^{\perp}$ by assumption, there exists a $\operatorname{Hom}_{\mathscr{A}}(\mathscr{C},-)$-exact exact sequence

$$
0 \rightarrow K_{n} \rightarrow C_{n-1} \rightarrow C_{n-2} \rightarrow \cdots \rightarrow C_{0} \stackrel{f_{0}}{\rightarrow} A \rightarrow 0
$$

in $\mathscr{A}$ with all $C_{i}$ 's objects in $\mathscr{C}$ and $K_{n}$ an object in $\mathscr{T}$ by Theorem 3.6. Note that both 


$$
0 \rightarrow A_{2} \stackrel{\left(\begin{array}{c}
0 \\
1_{A_{2}}
\end{array}\right)}{\longrightarrow} A \stackrel{\left(1_{A_{1}}, 0\right)}{\longrightarrow} A_{1} \rightarrow 0
$$

and

$$
0 \rightarrow A_{1} \stackrel{\left(\begin{array}{c}
1_{A_{1}} \\
0
\end{array}\right)}{\longrightarrow} A \stackrel{\left(0,1_{A_{2}}\right)}{\longrightarrow} A_{2} \rightarrow 0
$$

are exact and split. So both

$$
C_{0} \stackrel{\left(1_{\left.A_{1}, 0\right)} f_{0}\right.}{\longrightarrow} A_{1} \rightarrow 0
$$

and

$$
C_{0} \stackrel{\left(0,1_{A_{2}}\right) f_{0}}{\longrightarrow} A_{2} \rightarrow 0
$$

are $\operatorname{Hom}_{\mathscr{A}}(\mathscr{C},-)$-exact exact sequences. By [Hu, Theorem 3.6], we get the following $\operatorname{Hom}_{\mathscr{A}}(\mathscr{C},-)$-exact exact sequences:

$$
C_{0} \oplus C_{1} \rightarrow C_{0} \rightarrow A_{1} \rightarrow 0
$$

and

$$
C_{0} \oplus C_{1} \rightarrow C_{0} \rightarrow A_{2} \rightarrow 0
$$

Again by [Hu, Theorem 3.6], we get the following $\operatorname{Hom}_{\mathscr{A}}(\mathscr{C},-)$-exact exact sequences:

$$
C_{0} \oplus C_{1} \oplus C_{2} \rightarrow C_{0} \oplus C_{1} \rightarrow C_{0} \rightarrow A_{1} \rightarrow 0
$$

and

$$
C_{0} \oplus C_{1} \oplus C_{2} \rightarrow C_{0} \oplus C_{1} \rightarrow C_{0} \rightarrow A_{2} \rightarrow 0 .
$$

Continuing this procedure, we finally get the following $\operatorname{Hom}_{\mathscr{A}}(\mathscr{C},-)$-exact exact sequences:

$$
0 \rightarrow X_{n} \rightarrow \bigoplus_{i=0}^{n-1} C_{i} \rightarrow \bigoplus_{i=0}^{n-2} C_{i} \rightarrow \cdots \rightarrow C_{1} \oplus C_{0} \rightarrow C_{0} \rightarrow A_{1} \rightarrow 0
$$

and

$$
0 \rightarrow Y_{n} \rightarrow \bigoplus_{i=0}^{n-1} C_{i} \rightarrow \bigoplus_{i=0}^{n-2} C_{i} \rightarrow \cdots \rightarrow C_{1} \oplus C_{0} \rightarrow C_{0} \rightarrow A_{2} \rightarrow 0
$$

Put $U_{j}=\bigoplus_{i=0}^{j} C_{i}$ for any $0 \leq j \leq n-1$. Then we get a $\operatorname{Hom}_{\mathscr{A}}(\mathscr{C},-)$-exact exact sequence

$$
\begin{aligned}
0 & \rightarrow X_{n} \oplus Y_{n} \rightarrow U_{n-1} \oplus U_{n-1} \rightarrow U_{n-2} \oplus U_{n-2} \rightarrow \cdots \\
& \rightarrow U_{1} \oplus U_{1} \rightarrow U_{0} \oplus U_{0} \rightarrow A \rightarrow 0 .
\end{aligned}
$$

By Theorem 3.8, $X_{n} \oplus Y_{n}$ is an object in $\mathscr{T}$. So both $X_{n}$ and $Y_{n}$ are objects in $\mathscr{T}$ and hence $\mathscr{T}-\operatorname{dim} A_{1} \leq n$ and $\mathscr{T}-\operatorname{dim} A_{2} \leq n$.

The following result gives some sufficient conditions such that the $\mathscr{T}$-dimension and the $\mathscr{C}$-dimension of an object in $\mathscr{A}$ are identical. 
THEOREM 3.10

Let $\mathscr{T} \subseteq \mathscr{C}^{\perp} \cap^{\perp} \mathscr{C}$, and let $\mathscr{C}$ be closed under direct summands. Then for an object $A$ in $\mathscr{A}, \mathscr{T}-\operatorname{dim} A=\mathscr{C}-\operatorname{dim} A$ if one of the following conditions is satisfied.

(a) $\mathscr{C}-\operatorname{dim} A<\infty, \mathscr{E}=\mathscr{C}$, and $\mathscr{T}$ is closed under kernels of $\mathscr{C}$-proper epimorphisms.

(b) $\mathscr{C}-\operatorname{dim} A<\infty, \mathscr{E}=\mathscr{C}$, and $\mathscr{C}-\operatorname{dim}^{<\infty}$ is closed under direct summands.

(c) $A$ is an object in $\mathscr{T}^{\perp}$ and $\mathscr{C}$ is a cogenerator for $\mathscr{T}$.

\section{Proof}

It is trivial that $\mathscr{C}-\operatorname{dim} A \geq \mathscr{T}-\operatorname{dim} A$. In the following we prove that $\mathscr{C}-\operatorname{dim} A \leq$ $\mathscr{T}-\operatorname{dim} A$. Suppose that $\mathscr{T}-\operatorname{dim} A=n<\infty$.

(a) Let $\mathscr{C}-\operatorname{dim} A=t(<\infty)$. If $n<t$, then consider the following $\operatorname{Hom}_{\mathscr{A}}(\mathscr{C},-)-$ exact exact sequence:

$$
0 \rightarrow C_{t} \rightarrow \cdots \rightarrow C_{n} \rightarrow C_{n-1} \rightarrow \cdots \rightarrow C_{1} \rightarrow C_{0} \rightarrow A \rightarrow 0,
$$

in $\mathscr{A}$ with all $C_{i}$ 's objects in $\mathscr{C}$. Put $K_{n}=\operatorname{Im}\left(C_{n} \rightarrow C_{n-1}\right)$. So $K_{n}$ is an object in $\mathscr{T}$ by Theorem 3.8(a) and hence an object in $\perp_{\mathscr{C}}$ by assumption. This yields that the exact sequence

$$
0 \rightarrow C_{t} \rightarrow \cdots \rightarrow C_{n} \rightarrow K_{n} \rightarrow 0
$$

splits and $K_{n}$ is isomorphic to a direct summand of $C_{n}$. Because $\mathscr{C}$ is closed under direct summands by assumption, $K_{n}$ is an object in $\mathscr{C}$ and $\mathscr{C}-\operatorname{dim} A \leq n$, which is a contradiction. So $n \geq t$.

In the following, we prove (b) and (c).

Let

$$
0 \rightarrow T_{n} \rightarrow T_{n-1} \rightarrow \cdots \rightarrow T_{1} \rightarrow T_{0} \rightarrow A \rightarrow 0
$$

be an exact sequence in $\mathscr{A}$ with all $T_{i}$ 's objects in $\mathscr{T}$. By Proposition 3.4, we get an exact sequence

$$
0 \rightarrow K_{n} \rightarrow C_{n-1} \rightarrow \cdots \rightarrow C_{1} \rightarrow C_{0} \rightarrow A \rightarrow 0
$$

and a $\operatorname{Hom}_{\mathscr{A}}(\mathscr{E},-)$-exact exact sequence

$$
0 \rightarrow T \rightarrow K_{n} \rightarrow T_{n} \rightarrow 0
$$

in $\mathscr{A}$ with all $C_{i}$ 's objects in $\mathscr{C}$ and $T$ an object in $\mathscr{T}$. So $K_{n}$ is an object in $\mathscr{T}\left(\subseteq \mathscr{C}^{\perp} \cap{ }^{\perp} \mathscr{C}\right)$.

(b) Because $\mathscr{C}-\operatorname{dim} A<\infty, \mathscr{C}-\operatorname{dim} K_{n}<\infty$ by Lemma 2.4. By assumption $\mathscr{T} \subseteq{ }^{\perp} \mathscr{C}$, so it is easy to see that $K_{n}$ is isomorphic to a direct summand of some object in $\mathscr{C}$. Since $\mathscr{C}$ is closed under direct summands by assumption, $K_{n}$ is an object in $\mathscr{C}$ and $\mathscr{C}-\operatorname{dim} A \leq n$.

(c) Let $A$ be an object in $\mathscr{T}^{\perp}$, and let $K_{i}=\operatorname{Im}\left(C_{i} \rightarrow C_{i-1}\right)$ for any $1 \leq i \leq$ $n-1$. Then all $K_{i}$ 's are objects in $\mathscr{C}^{\perp}$. By Lemma 2.6, all $K_{i}$ 's are objects in $\mathscr{T}^{\perp}$, and in particular, $K_{n}$ is an object in $\mathscr{T}^{\perp}$. So $K_{n}$ is an object in $\mathscr{C}$ by Lemma 2.7, and hence $\mathscr{C}-\operatorname{dim} A \leq n$. 
The following result gives a sufficient condition such that the $\mathscr{T}$-codimension and the $\mathscr{C}$-codimension of an object in $\mathscr{A}$ are identical.

THEOREM 3.11

Let $\mathscr{D}$ be a subcategory of $\mathscr{A}$ such that $\mathscr{T} \subseteq{ }^{\perp} \mathscr{C} \cap \mathscr{D}^{\perp}$, and let $\mathscr{C}$-codim $\leq n$ be closed under direct summands for any $n \geq 0$. If $M$ is an object in $\mathscr{A}$ with $\mathscr{D}-\operatorname{dim} M<\infty$, then $\mathscr{T}-\operatorname{codim} M=\mathscr{C}-\operatorname{codim} M$.

Proof

It is clear that $\mathscr{C}$-codim $M \geq \mathscr{T}$-codim $M$. In the following we prove that $\mathscr{T}$-codim $M \geq \mathscr{C}$-codim $M$.

Without loss of generality, assume that $\mathscr{T}$-codim $M=n<\infty$. If $n=0$, then $M$ is an object in $\mathscr{T}$ and there exists a $\operatorname{Hom}_{\mathscr{A}}(\mathscr{E},-)$-exact exact sequence

$$
0 \rightarrow M^{\prime} \rightarrow C \rightarrow M \rightarrow 0
$$

in $\mathscr{A}$ with $C$ an object in $\mathscr{C}$ and $M^{\prime}$ an object in $\mathscr{T}$. Notice that $\mathscr{T} \subseteq{ }^{\perp} \mathscr{C} \cap \mathscr{D}^{\perp}$ by assumption, so $\operatorname{Ext}_{\mathscr{A}}^{i}\left(M, M^{\prime}\right)=0$ for any $i \geq 1$ by Lemma 2.2. It follows that the exact sequence (3.3) splits, which implies that $M$ is isomorphic to a direct summand of $C$. Because $\mathscr{C}$ is closed under direct summands by assumption, $M$ is an object in $\mathscr{C}$.

Now suppose that $n \geq 1$. By Theorem 3.7, there exists an exact sequence

$$
0 \rightarrow M \rightarrow T \rightarrow H \rightarrow 0
$$

in $\mathscr{A}$ with $T$ an object in $\mathscr{T}$ and $\mathscr{C}$-codim $H \leq n-1$. It is easy to see that $M$ is an object in $\perp_{\mathscr{C}}$. Because there exists a $\operatorname{Hom}_{\mathscr{A}}(\mathscr{E},-)$-exact exact sequence

$$
0 \rightarrow T^{\prime} \rightarrow C^{\prime} \rightarrow T \rightarrow 0
$$

in $\mathscr{A}$ with $C^{\prime}$ an object in $\mathscr{C}$ and $T^{\prime}$ an object in $\mathscr{T}$, we have the following pullback diagram:

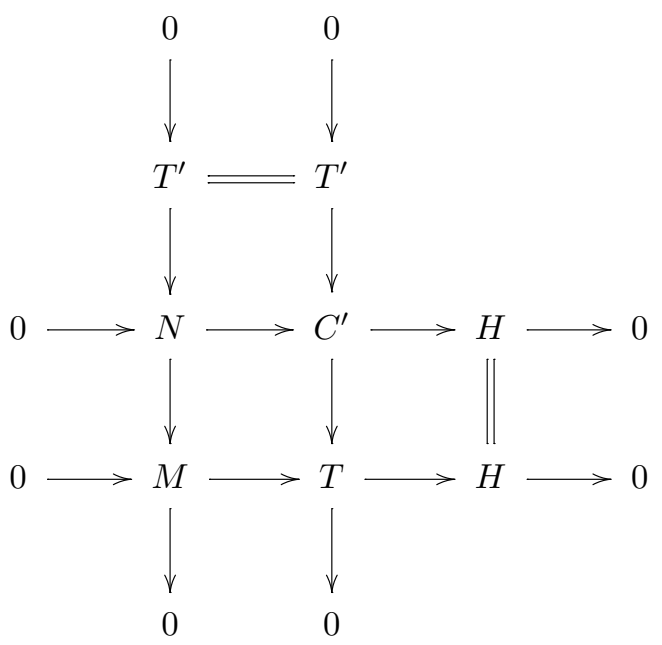


By the exactness of the middle row in the above diagram, $\mathscr{C}$-codim $N \leq n$. Because $\operatorname{Ext}_{\mathscr{A}}^{1}\left(M, T^{\prime}\right)=0$ by Lemma 2.2, the first column in the above diagram splits. So $M$ is isomorphic to a direct summand of $N$. Because $\mathscr{C}$-codim ${ }^{\leq n}$ is closed under direct summands by assumption, $\mathscr{C}$-codim $M \leq n$.

In the following, we fix a subcategory $\mathscr{C}$ of $\mathscr{A}$.

The following two corollaries give some sufficient conditions such that the $\mathcal{G}(\mathscr{C})$-dimension and the $\mathscr{C}$-dimension of an object in $\mathscr{A}$ are identical. The first one is a generalization of [Z, Theorem 2.3].

\section{COROLLARY 3.12}

Let $\mathscr{C} \perp \mathscr{C}$, and let $\mathscr{C}$ be closed under direct summands. Then for any object $A$ in $\mathcal{G}(\mathscr{C})^{\perp}, \mathcal{G}(\mathscr{C})-\operatorname{dim} A=\mathscr{C}-\operatorname{dim} A$.

\section{Proof}

Let $\mathscr{C} \perp \mathscr{C}$. It is clear that $\mathscr{C}$ is a $\mathscr{C}$-proper generator and a $\mathscr{C}$-coproper cogenerator for $\mathcal{G}(\mathscr{C})$. By [SSW, Corollary 4.5], $\mathcal{G}(\mathscr{C})$ is closed under extensions. By [Hu, Lemma 5.7], $\mathcal{G}(\mathscr{C}) \subseteq \mathscr{C}^{\perp} \cap{ }^{\perp} \mathscr{C}$. Now the assertion follows from Theorem $3.10(\mathrm{c})$.

The following is a generalization of [H2, Theorem 2.2] and [Z, Corollary 2.5].

\section{COROLLARY 3.13}

Let $\mathscr{C} \perp \mathscr{C}$, let $\mathscr{C}$ be closed under direct summands, and let $\mathscr{D}$ be a subcategory of $\mathcal{G}(\mathscr{C})^{\perp}$. Then for any object $A$ in $\mathscr{C}^{\perp}$ with $\mathscr{D}-\operatorname{codim} A<\infty, \mathcal{G}(\mathscr{C})-\operatorname{dim} A=$ $\mathscr{C}-\operatorname{dim} A$.

\section{Proof}

Let $A$ be an object in $\mathscr{C}^{\perp}$ with $\mathscr{D}$-codim $A<\infty$. Because $\mathscr{D}$ is a subcategory of $\mathcal{G}(\mathscr{C})^{\perp}$, it is easy to see that $A$ is an object in $\mathcal{G}(\mathscr{C})^{\perp}$ by Lemma 2.6. Then the assertion follows from Corollary 3.12.

The following result gives a sufficient condition such that the $\mathcal{G}(\mathscr{C})$-dimension and the ${ }^{\perp} \mathscr{C}$-dimension of an object in $\mathscr{A}$ are identical.

\section{THEOREM 3.14}

Let $\mathscr{C} \perp \mathscr{C}$, and let $A$ an object in $\mathscr{A}$ with $\mathcal{G}(\mathscr{C})-\operatorname{dim} A<\infty$. Then $\mathcal{G}(\mathscr{C})-\operatorname{dim} A=$ $\perp \mathscr{C}-\operatorname{dim} A$.

\section{Proof}

By [Hu, Lemma 5.7], $\mathcal{G}(\mathscr{C})-\operatorname{dim} A \geq{ }^{\perp} \mathscr{C}-\operatorname{dim} A$. In the following we prove that $\mathcal{G}(\mathscr{C})-\operatorname{dim} A \leq{ }^{\perp} \mathscr{C}-\operatorname{dim} A$.

Suppose that ${ }^{\perp} \mathscr{C}-\operatorname{dim} A=n<\infty$ and that $\mathcal{G}(\mathscr{C})-\operatorname{dim} A=m<\infty$. If $n=0$, then $A$ is an object in ${ }^{\perp} \mathscr{C}$. So by [Hu, Theorem 5.8], $A$ is an object in $\mathcal{G}(\mathscr{C})$ 
and $m=0$. Let $n \geq 1$. Then $\operatorname{Ext}_{\mathscr{A}}^{n+i}(A, C)=0$ for any object $C$ in $\mathscr{C}$ and $i \geq 1$. If $m>n$, then consider the following exact sequence:

$$
0 \rightarrow G_{m} \rightarrow \cdots \rightarrow G_{n} \rightarrow G_{n-1} \rightarrow G_{1} \rightarrow G_{0} \rightarrow A \rightarrow 0,
$$

in $\mathscr{A}$ with all $G_{i}$ 's objects in $\mathcal{G}(\mathscr{C})$. By putting $K_{n}=\operatorname{Im}\left(G_{n} \rightarrow G_{n-1}\right), K_{n}$ is an object in ${ }^{\perp} \mathscr{C}$ and $\mathcal{G}(\mathscr{C})-\operatorname{dim} K_{n} \leq m-n<\infty$. By the above argument, $K_{n}$ is an object in $\mathcal{G}(\mathscr{C})$. So $\mathcal{G}(\mathscr{C})-\operatorname{dim} A \leq n$, which is a contradiction. Thus $m \leq n$. It follows that $\mathcal{G}(\mathscr{C})-\operatorname{dim} A \leq{ }^{\perp} \mathscr{C}-\operatorname{dim} A$.

\section{Dual results}

In this section, we introduce the notion of (pre)coresolving subcategories of $\mathscr{A}$. Then we give some criteria for computing and comparing homological codimensions relative to different precoresolving subcategories. The results and their proofs in this section are completely dual to those in Section 3, so we only list the results without proofs.

\section{DEFINITION 4.1}

Let $\mathscr{E}$ and $\mathscr{T}$ be subcategories of $\mathscr{A}$. Then $\mathscr{T}$ is called $\mathscr{E}$-precoresolving in $\mathscr{A}$ if the following conditions are satisfied.

(a) $\mathscr{T}$ admits an $\mathscr{E}$-coproper cogenerator.

(b) $\mathscr{T}$ is closed under $\mathscr{E}$-coproper extensions, that is, for any $\operatorname{Hom}_{\mathscr{A}}(-, \mathscr{E})$ exact exact sequence $0 \rightarrow A_{1} \rightarrow A_{2} \rightarrow A_{3} \rightarrow 0$ in $\mathscr{A}$, if both $A_{1}$ and $A_{3}$ are objects in $\mathscr{T}$, then $A_{2}$ is also an object in $\mathscr{T}$.

An $\mathscr{E}$-precoresolving subcategory $\mathscr{T}$ of $\mathscr{A}$ is called $\mathscr{E}$-coresolving if the following condition is satisfied.

(c) $\mathscr{T}$ is closed under cokernels of $\mathscr{E}$-coproper monomorphisms, that is, for any $\operatorname{Hom}_{\mathscr{A}}(-, \mathscr{E})$-exact exact sequence $0 \rightarrow A_{1} \rightarrow A_{2} \rightarrow A_{3} \rightarrow 0$ in $\mathscr{A}$, if both $A_{1}$ and $A_{2}$ are objects in $\mathscr{T}$, then $A_{3}$ is also an object in $\mathscr{T}$.

The following list shows that the class of $\mathscr{E}$-(pre)coresolving subcategories is rather large.

\section{EXAMPLE 4.2}

(a) Let $\mathscr{A}$ admit enough injective objects, and let $\mathscr{E}$ be the subcategory of $\mathscr{A}$ consisting of injective objects. Then a subcategory of $\mathscr{A}$ closed under $\mathscr{E}$-coproper extensions is just a subcategory of $\mathscr{A}$ closed under extensions. Furthermore, if $\mathscr{C}=\mathscr{E}$ in the above definition, then an $\mathscr{E}$-precoresolving subcategory is just a subcategory which contains all injective objects and is closed under extensions, and an $\mathscr{E}$-coresolving subcategory is just an injectively coresolving subcategory in the sense of [H1].

(b) Let $\mathscr{C}$ be a subcategory of $\mathscr{A}$ with $\mathscr{C} \perp \mathscr{C}$. Then by [SSW, Corollary 4.5], the Gorenstein subcategory $\mathcal{G}(\mathscr{C})$ of $\mathscr{A}$ is a $\mathscr{C}$-precoresolving subcategory of $\mathscr{A}$ 
with a $\mathscr{C}$-coproper cogenerator $\mathscr{C}$; furthermore, if $\mathscr{C}$ is closed under cokernels of monomorphisms, then $\mathcal{G}(\mathscr{C})$ is a $\mathscr{C}$-coresolving subcategory of $\mathscr{A}$ by [SSW, Theorem 4.12(b)].

(c) Let $R$ be a ring, and let $\mathcal{I}(\operatorname{Mod} R)$ be the subcategory of $\operatorname{Mod} R$ consisting of injective modules. If $(\mathscr{X}, \mathscr{Y})$ is a cotorsion pair in $\operatorname{Mod} R$, then $\mathscr{Y}$ is an $\mathcal{I}(\operatorname{Mod} R)$-precoresolving subcategory of $\operatorname{Mod} R$ with an $\mathcal{I}(\operatorname{Mod} R)$-coproper cogenerator $\mathcal{I}(\operatorname{Mod} R)$ (see [EJ2]).

(d) Let $R$ be a ring. Recall that a module $E$ in $\operatorname{Mod} R$ is called FP-injective if $\operatorname{Ext}_{R}^{1}(M, E)=0$ for any finitely presented left $R$-module $M$. The FP-injective modules are also known as absolutely pure modules. We use $\mathcal{F} \mathcal{I}(\operatorname{Mod} R)$ to denote the subcategory of $\operatorname{Mod} R$ consisting of FP-injective modules. Then by [Hu, Lemma 3.1 and Theorem 3.4], it is not difficult to see that the subcategory of $\operatorname{Mod} R$ consisting of Gorenstein FP-injective modules (see [MD] or Section 5 below for the definition) is an $\mathcal{F} \mathcal{I}(\operatorname{Mod} R)$-coresolving subcategory of $\operatorname{Mod} R$ with an $\mathcal{F} \mathcal{I}(\operatorname{Mod} R)$-coproper cogenerator $\mathcal{I}(\operatorname{Mod} R)$.

(e) Let $R$ be a ring. We denote by cores $\mathcal{P}(\widetilde{\operatorname{Mod}} R)=\{M \in \operatorname{Mod} R \mid$ there exists a $\operatorname{Hom}_{R}(-, \mathcal{P}(\operatorname{Mod} R))$-exact exact sequence $0 \rightarrow M \rightarrow P^{0} \rightarrow P^{1} \rightarrow \cdots \rightarrow$ $P^{i} \rightarrow \cdots$ in $\operatorname{Mod} R$ with all $P^{i}$ s projective . Then by [Hu, Lemma 3.1 and Theorem 3.8], it is easy to see that cores $\mathcal{P}(\widehat{\operatorname{Mod} R} R)$ is a $\mathcal{P}(\operatorname{Mod} R)$-coresolving subcategory of $\operatorname{Mod} R$ with a $\mathcal{P}(\operatorname{Mod} R)$-coproper cogenerator $\mathcal{P}(\operatorname{Mod} R)$. Let $R$ be a left and right Noetherian ring. Then by [AB, Theorem 2.17] and [Hu, Lemma 3.1], the subcategory of $\bmod R$ consisting of $\infty$-torsion-free modules (see $[\mathrm{HuH}]$ or Section 5 below for the definition $)$ is a $\mathcal{P}(\bmod R)$-coresolving subcategory of $\bmod R$ with a $\mathcal{P}(\bmod R)$-coproper cogenerator $\mathcal{P}(\bmod R)$.

Unless stated otherwise, in the rest of this section, we fix a subcategory $\mathscr{E}$ of $\mathscr{A}$ and an $\mathscr{E}$-precoresolving subcategory $\mathscr{T}$ of $\mathscr{A}$ admitting an $\mathscr{E}$-coproper cogenerator $\mathscr{C}$. We will give some criteria for computing the $\mathscr{T}$-codimension of a given object $A$ in $\mathscr{A}$, and then compare it with the $\mathscr{C}$-codimension of $A$.

The following two propositions play a crucial role in this section.

\section{PROPOSITION 4.3}

Let

$$
0 \rightarrow M \rightarrow T^{0} \rightarrow T^{1} \rightarrow A \rightarrow 0
$$

be an exact sequence in $\mathscr{A}$ with both $T^{0}$ and $T^{1}$ objects in $\mathscr{T}$. Then we have the following.

(a) There exists an exact sequence

$$
0 \rightarrow M \rightarrow C \rightarrow T \rightarrow A \rightarrow 0
$$

in $\mathscr{A}$ with $T$ an object in $\mathscr{T}$ and $C$ an object in $\mathscr{C}$.

(b) If (4.1) is $\operatorname{Hom}_{\mathscr{A}}(-, X)$-exact for some object $X$ in $\mathscr{A}$, then so is (4.2).

As an application of Proposition 4.3, we get the following. 
PROPOSITION 4.4

Let $n \geq 1$, and let

$$
0 \rightarrow M \rightarrow T^{0} \rightarrow T^{1} \rightarrow \cdots \rightarrow T^{n-1} \rightarrow A \rightarrow 0
$$

be an exact sequence in $\mathscr{A}$ with all $T^{i}$ 's objects in $\mathscr{T}$. Then there exist an exact sequence

$$
0 \rightarrow M \rightarrow C^{0} \rightarrow C^{1} \rightarrow \cdots \rightarrow C^{n-1} \rightarrow B \rightarrow 0
$$

and a $\operatorname{Hom}_{\mathscr{A}}(-, \mathscr{E})$-exact exact sequence

$$
0 \rightarrow A \rightarrow B \rightarrow T \rightarrow 0
$$

in $\mathscr{A}$ with all $C^{i}$ 's objects in $\mathscr{C}$ and $T$ an object in $\mathscr{T}$. In particular, an object in $\mathscr{A}$ is an $n-\mathscr{C}$-syzygy if and only if it is an $n-\mathscr{T}$-syzygy.

The following corollary is an immediate consequence of Proposition 4.4.

\section{COROLLARY 4.5}

Let $A$ be an object in $\mathscr{A}$, and let $n \geq 0$. If $\mathscr{T}-\operatorname{dim} A=n$, then there exists a $\operatorname{Hom}_{\mathscr{A}}(-, \mathscr{E})$-exact exact sequence $0 \rightarrow A \rightarrow B \rightarrow T \rightarrow 0$ in $\mathscr{A}$ with $\mathscr{C}$ - $\operatorname{dim} B \leq n$ and $T$ an object in $\mathscr{T}$.

We give a criterion for computing the $\mathscr{T}$-codimension of an object in $\mathscr{A}$ as follows.

\section{THEOREM 4.6}

The following statements are equivalent for any object $M$ in $\mathscr{A}$ and $n \geq 0$.

(a) $\mathscr{T}$-codim $M \leq n$.

(b) There exists an exact sequence

$$
0 \rightarrow M \rightarrow C^{0} \rightarrow C^{1} \rightarrow \cdots \rightarrow C^{n-1} \rightarrow K^{n} \rightarrow 0
$$

in $\mathscr{A}$ with all $C^{i}$ 's objects in $\mathscr{C}$ and $K^{n}$ an object in $\mathscr{T}$.

The following result gives a criterion for computing the $\mathscr{T}$-dimension of an object in $\mathscr{A}$.

\section{THEOREM 4.7}

The following statements are equivalent for any object $A$ in $\mathscr{A}$ and $n \geq 0$.

(a) $\mathscr{T}-\operatorname{dim} A \leq n$.

(b) There exists an exact sequence

$$
0 \rightarrow C_{n} \rightarrow C_{n-1} \rightarrow \cdots \rightarrow C_{1} \rightarrow K_{0} \rightarrow A \rightarrow 0
$$

in $\mathscr{A}$ with $K_{0}$ an object in $\mathscr{T}$ and all $C_{i}$ 's objects in $\mathscr{C}$; that is, there exists an exact sequence

$$
0 \rightarrow H \rightarrow T \rightarrow A \rightarrow 0
$$

in $\mathscr{A}$ with $T$ an object in $\mathscr{T}$ and $\mathscr{C}-\operatorname{dim} H \leq n-1$. 
The following result gives a sufficient condition such that the $n$ - $\mathscr{C}$-cosyzygy of an object in $\mathscr{A}$ with $\mathscr{T}$-codimension at most $n$ is in $\mathscr{T}$.

\section{THEOREM 4.8}

Let $\mathscr{T}$ be closed under cokernels of (E्E-coproper) monomorphisms and $\mathscr{T} \subseteq{ }^{\perp} \mathscr{C}$, and let $n \geq 0$. Then for any object $M$ in $\mathscr{A}$ with $\mathscr{T}$-codim $M \leq n$ we have the following.

(a) For any $\left(\operatorname{Hom}_{\mathscr{A}}(-, \mathscr{E})\right.$-exact) exact sequence $0 \rightarrow M \rightarrow C^{0} \rightarrow C^{1} \rightarrow$ $\cdots \rightarrow C^{n-1} \rightarrow K^{n} \rightarrow 0$ in $\mathscr{A}$ with all $C^{i}$ 's objects in $\mathscr{C}, K^{n}$ is an object in $\mathscr{T}$.

(b) If $0 \rightarrow M \rightarrow C \rightarrow K \rightarrow 0$ is a $\left(\operatorname{Hom}_{\mathscr{A}}(-, \mathscr{E})\right.$-exact) exact sequence in $\mathscr{A}$ with $C$ an object in $\mathscr{C}$, then $\mathscr{T}-\operatorname{codim} K \leq n-1$.

We use $\mathscr{T}$-codim ${ }^{\leq n}$ to denote the subcategory of $\mathscr{A}$ consisting of objects with $\mathscr{T}$-codimension at most $n$.

\section{COROLLARY 4.9}

Let $\mathscr{T}$ be a $\mathscr{C}$-coresolving subcategory of $\mathscr{A}$ with a $\mathscr{C}$-coproper cogenerator $\mathscr{C}$ and $\mathscr{T} \subseteq{ }^{\perp} \mathscr{C}$. If $\mathscr{T}$ is closed under direct summands, then so is $\mathscr{T}$-codim ${ }^{\leq n}$ for any $n \geq 0$.

The following result gives some sufficient conditions such that the $\mathscr{T}$-codimension and the $\mathscr{C}$-codimension of an object in $\mathscr{A}$ are identical.

\section{THEOREM 4.10}

Let $\mathscr{T} \subseteq \mathscr{C}^{\perp} \cap{ }^{\perp} \mathscr{C}$, and let $\mathscr{C}$ be closed under direct summands. Then for an object $M$ in $\mathscr{A}, \mathscr{T}$-codim $M=\mathscr{C}$-codim $M$ if one of the following conditions is satisfied.

(a) $\mathscr{C}$-codim $M<\infty, \mathscr{E}=\mathscr{C}$, and $\mathscr{T}$ is closed under cokernels of $\mathscr{C}$-coproper monomorphisms.

(b) $\mathscr{C}-\operatorname{codim} M<\infty, \mathscr{E}=\mathscr{C}$, and $\mathscr{C}-\operatorname{dim}^{<\infty}$ is closed under direct summands.

(c) $M$ is an object in $\perp \mathscr{T}$ and $\mathscr{C}$ is a generator for $\mathscr{T}$.

The following result gives a sufficient condition such that the $\mathscr{T}$-dimension and the $\mathscr{C}$-dimension of an object in $\mathscr{A}$ are identical.

\section{THEOREM 4.11}

Let $\mathscr{D}$ be a subcategory of $\mathscr{A}$ such that $\mathscr{T} \subseteq \mathscr{C}^{\perp} \cap \cap^{\perp} \mathscr{D}$, and let $\mathscr{C}-\operatorname{dim}{ }^{\leq n}$ be closed under direct summands for any $n \geq 0$. If $A$ is an object in $\mathscr{A}$ with $\mathscr{D}$-codim $A<\infty$, then $\mathscr{T}-\operatorname{dim} A=\mathscr{C}-\operatorname{dim} A$.

In the following, we fix a subcategory $\mathscr{C}$ of $\mathscr{A}$. 
The following two corollaries give some sufficient conditions such that the $\mathcal{G}(\mathscr{C})$-codimension and the $\mathscr{C}$-codimension of an object in $\mathscr{A}$ are identical.

COROLLARY 4.12

Let $\mathscr{C} \perp \mathscr{C}$, and let $\mathscr{C}$ be closed under direct summands. Then for any object $M$ in ${ }^{\perp} \mathcal{G}(\mathscr{C}), \mathcal{G}(\mathscr{C})$-codim $M=\mathscr{C}$-codim $M$.

\section{COROLLARY 4.13}

Let $\mathscr{C} \perp \mathscr{C}$, let $\mathscr{C}$ be closed under direct summands, and let $\mathscr{D}$ be a subcategory of ${ }^{\perp} \mathcal{G}(\mathscr{C})$. Then for any object $M$ in ${ }^{\perp} \mathscr{C}$ with $\mathscr{D}-\operatorname{dim} M<\infty, \mathcal{G}(\mathscr{C})-\operatorname{codim} M=$ $\mathscr{C}-\operatorname{codim} M$.

The following result gives a sufficient condition such that the $\mathcal{G}(\mathscr{C})$-codimension and the $\mathscr{C}^{\perp}$-codimension of an object in $\mathscr{A}$ are identical.

\section{THEOREM 4.14}

Let $\mathscr{C} \perp \mathscr{C}$, and let $M$ be an object in $\mathscr{A}$ with $\mathcal{G}(\mathscr{C})$-codim $M<\infty$. Then $\mathcal{G}(\mathscr{C})-\operatorname{codim} M=\mathscr{C}^{\perp}-\operatorname{codim} M$.

\section{Applications and questions}

In this section, we will apply the results in Sections 3 and 4 to special subcategories and in particular to module categories. Finally, we propose some open questions and conjectures concerning the obtained results.

\subsection{Special subcategories}

We define res $\widetilde{\mathscr{C}}=\left\{A\right.$ is an object in $\mathscr{A} \mid$ there exists a $\operatorname{Hom}_{\mathscr{A}}(\mathscr{C},-)$-exact exact sequence $\cdots \rightarrow C_{i} \rightarrow \cdots \rightarrow C_{1} \rightarrow C_{0} \rightarrow A \rightarrow 0$ in $\mathscr{A}$ with all $C_{i}$ 's objects in $\left.\mathscr{C}\right\}$. Dually, we define cores $\tilde{\mathscr{C}}=\left\{M\right.$ is an object in $\mathscr{A} \mid$ there exists a $\operatorname{Hom}_{\mathscr{A}}(-, \mathscr{C})$ exact exact sequence $0 \rightarrow M \rightarrow C^{0} \rightarrow C^{1} \rightarrow \cdots \rightarrow C^{i} \rightarrow \cdots$ in $\mathscr{A}$ with all $C^{i}$, s objects in $\mathscr{C}\}$ (see [SSW]).

We have the following.

\section{FACT 5.1}

(a) Note that $\mathscr{C}$ is a $\mathscr{C}$-proper generator for res $\widetilde{\mathscr{C}}$ and $\operatorname{res} \widetilde{\mathscr{C}} \cap \perp \mathscr{C}$. By $[\mathrm{Hu}$, Lemma 3.1(1)], both res $\widetilde{\mathscr{C}}$ and res $\widetilde{\mathscr{C}} \cap \perp \mathscr{C}$ are closed under $\mathscr{C}$-proper extensions. So both res $\widetilde{\mathscr{C}}$ and res $\widetilde{\mathscr{C}} \cap{ }^{\perp} \mathscr{C}$ are $\mathscr{C}$-preresolving. We remark that if $\mathscr{C}$ is a $\mathscr{C}$ proper generator for $\mathscr{A}$, then res $\widetilde{\mathscr{C}}=\mathscr{A}$ and res $\widetilde{\mathscr{C}} \cap \perp \mathscr{C}={ }^{\perp} \mathscr{C}$.

(b) If $\mathscr{C}$ is closed under kernels of epimorphisms, then so are both res $\widetilde{\mathscr{C}}$ and $\operatorname{res} \tilde{\mathscr{C}} \cap \perp \mathscr{C}$ (see [Hu, Proposition $4.7(1)]$ ).

Dually, we have the following.

(c) Note that $\mathscr{C}$ is a $\mathscr{C}$-coproper cogenerator for cores $\widetilde{\mathscr{C}}$ and $\mathscr{C}^{\perp} \cap \operatorname{cores} \widetilde{\mathscr{C}}$. By [Hu, Lemma 3.1(2)], both cores $\tilde{\mathscr{C}}$ and $\mathscr{C}^{\perp} \cap \operatorname{cores} \tilde{\mathscr{C}}$ are closed under $\mathscr{C}$ coproper extensions. So both cores $\widetilde{\mathscr{C}}$ and $\mathscr{C}^{\perp} \cap \operatorname{cores} \widetilde{\mathscr{C}}$ are $\mathscr{C}$-precoresolving. 
We also remark that if $\mathscr{C}$ is a $\mathscr{C}$-coproper cogenerator for $\mathscr{A}$, then cores $\widetilde{\mathscr{C}}=\mathscr{A}$ and $\mathscr{C}^{\perp} \cap \operatorname{cores} \widetilde{\mathscr{C}}=\mathscr{C}^{\perp}$.

(d) If $\mathscr{C}$ is closed under cokernels of monomorphisms, then so are both cores $\tilde{\mathscr{C}}$ and $\mathscr{C}^{\perp} \cap \operatorname{cores} \tilde{\mathscr{C}}$ (see [Hu, Proposition 4.7(2)]).

\section{APPLICATION 5.2}

By Fact 5.1, we can apply the results in Section 3 in the cases for $\mathscr{T}=\operatorname{res} \tilde{\mathscr{C}}$ and $\mathscr{T}=\operatorname{res} \tilde{\mathscr{C}} \cap{ }^{\perp} \mathscr{C}$, respectively, and apply the results in Section 4 in the cases for $\mathscr{T}=\operatorname{cores} \tilde{\mathscr{C}}$ and $\mathscr{T}=\mathscr{C}^{\perp} \cap \operatorname{cores} \widetilde{\mathscr{C}}$, respectively. We will not list these consequences in details.

\subsection{Module categories}

In this subsection, $R$ is a ring and all subcategories of $\operatorname{Mod} R$ are full and additive. For a module $A$ in $\operatorname{Mod} R$, we denote the projective, injective, and flat dimensions of $A$ by $\operatorname{pd}_{R} A, \operatorname{id}_{R} A$, and $\operatorname{fd}_{R} A$, respectively.

We first give the following.

\section{PROPOSITION 5.3}

Let $\mathscr{T}$ be a subcategory of $\operatorname{Mod} R$.

(a) If $\mathscr{T}$ is closed under extensions and $\mathcal{P}(\operatorname{Mod} R) \subseteq \mathscr{T} \subseteq{ }^{\perp} \mathcal{P}(\operatorname{Mod} R)$, then $\operatorname{pd}_{R} A=\mathscr{T}-\operatorname{dim} A$ for any $A \in \operatorname{Mod} R$ with $\operatorname{pd}_{R} A<\infty$.

(b) If $\mathscr{T}$ is closed under extensions and $\mathcal{I}(\operatorname{Mod} R) \subseteq \mathscr{T} \subseteq \mathcal{I}(\operatorname{Mod} R)^{\perp}$, then $\operatorname{id}_{R} A=\mathscr{T}$-codim $A$ for any $A \in \operatorname{Mod} R$ with $\operatorname{id}_{R} A<\infty$.

\section{Proof}

(a) Because $\mathscr{T} \subseteq{ }^{\perp} \mathcal{P}(\operatorname{Mod} R)=\mathcal{P}(\operatorname{Mod} R)^{\perp} \cap^{\perp} \mathcal{P}(\operatorname{Mod} R)$ by assumption, we get the assertion by Theorem $3.10(\mathrm{~b})$.

(b) It is dual to (a).

Let $(\mathscr{X}, \mathscr{Y})$ be a cotorsion pair in $\operatorname{Mod} R$. Then $\mathscr{X} \cap \mathscr{Y}$ is called the heart of $(\mathscr{X}, \mathscr{Y})$. A cotorsion pair $(\mathscr{X}, \mathscr{Y})$ is called hereditary if $\mathscr{X}={ }^{\perp} \mathscr{Y}$ and $\mathscr{Y}=\mathscr{X}^{\perp}$; in this case, $\mathscr{X}$ is projectively resolving and $\mathscr{Y}$ is injectively coresolving (see [GT, Lemma 2.2.10]). By Proposition 5.3 we immediately have the following.

\section{COROLLARY 5.4}

Let $(\mathscr{X}, \mathscr{Y})$ be a hereditary cotorsion pair in $\operatorname{Mod} R$ with the heart $\mathscr{C}$ $(=\mathscr{X} \cap \mathscr{Y})$.

(a) If $\mathscr{C}=\mathcal{P}(\operatorname{Mod} R)$, then for any $A \in \operatorname{Mod} R$ with $\operatorname{pd}_{R} A<\infty, \operatorname{pd}_{R} A=$ $\mathscr{X}-\operatorname{dim} A$.

(b) If $\mathscr{C}=\mathcal{I}(\operatorname{Mod} R)$, then for any $A \in \operatorname{Mod} R$ with $\operatorname{id}_{R} A<\infty, \operatorname{id}_{R} A=$ $\mathscr{Y}-\operatorname{codim} A$. 
Note that a module in $\mathcal{G}(\mathcal{P}(\operatorname{Mod} R)$ ) (resp., $\mathcal{G}(\mathcal{I}(\operatorname{Mod} R))$ ) is just a Gorenstein projective (resp., injective) module in $\operatorname{Mod} R$. So $\mathcal{G}(\mathcal{P}(\operatorname{Mod} R))-\operatorname{dim}_{R} A$ (resp., $\left.\mathcal{G}(\mathcal{I}(\operatorname{Mod} R))-\operatorname{codim}_{R} A\right)$ is just the Gorenstein projective (resp., injective) dimension of a module $A$ in $\operatorname{Mod} R$. We denote the Gorenstein projective (resp., injective) dimension of a module $A$ in $\operatorname{Mod} R$ by $\operatorname{Gpd}_{R} A$ (resp., $\left.\operatorname{Gid}_{R} A\right)$.

\section{DEFINITION 5.5}

Let $A$ be a module in $\operatorname{Mod} R$.

(a) ([DLM]) $A$ is called strongly Gorenstein flat if there exists a $\operatorname{Hom}_{R}(-$, $\mathcal{F}(\operatorname{Mod} R)$ )-exact exact sequence $\cdots \rightarrow P_{1} \rightarrow P_{0} \rightarrow P^{0} \rightarrow P^{1} \rightarrow \cdots$ in $\operatorname{Mod} R$ with all terms projective such that $A \cong \operatorname{Im}\left(P_{0} \rightarrow P^{0}\right)$. We use $\mathcal{S G \mathcal { F }}(\operatorname{Mod} R)$ to denote the subcategory of $\operatorname{Mod} R$ consisting of strongly Gorenstein flat modules.

The strongly Gorenstein flat dimension $\operatorname{SGfd}_{R} A$ of $A$ is defined to be $\inf \{n \mid$ there exists an exact sequence $0 \rightarrow G_{n} \rightarrow \cdots \rightarrow G_{1} \rightarrow G_{0} \rightarrow A \rightarrow 0$ in $\operatorname{Mod} R$ with all $G_{i}$ 's in $\left.\mathcal{S G \mathcal { F }}(\operatorname{Mod} R)\right\}$. Set $\operatorname{SGfd}_{R} A=\infty$ if no such $n$ exists.

(b) $([\mathrm{MD}]) A$ is called Gorenstein FP-injective if there exists a $\operatorname{Hom}_{R}(\mathcal{F} \mathcal{I}(\operatorname{Mod} R),-)$-exact exact sequence $\cdots \rightarrow I_{1} \rightarrow I_{0} \rightarrow I^{0} \rightarrow I^{1} \rightarrow \cdots$ in $\operatorname{Mod} R$ with all terms injective such that $A \cong \operatorname{Im}\left(I_{0} \rightarrow I^{0}\right)$. We use $\mathcal{G F} \mathcal{I}(\operatorname{Mod} R)$ to denote the subcategory of $\operatorname{Mod} R$ consisting of Gorenstein FP-injective modules.

The Gorenstein FP-injective dimension $\operatorname{GFid}_{R} A$ of $A$ is defined to be $\inf \{n \mid$ there exists an exact sequence $0 \rightarrow A \rightarrow H^{0} \rightarrow H^{1} \rightarrow \cdots \rightarrow H^{n} \rightarrow 0$ in $\operatorname{Mod} R$ with all $H^{i}$, s in $\left.\mathcal{G} \mathcal{F} \mathcal{I}(\operatorname{Mod} R)\right\}$. Set $\operatorname{GFid}_{A}=\infty$ if no such $n$ exists.

It is trivial that there exist the following inclusions:

$\mathcal{P}(\operatorname{Mod} R) \subseteq \mathcal{S G \mathcal { F }}(\operatorname{Mod} R)$

$$
\subseteq \mathcal{G}(\mathcal{P}(\operatorname{Mod} R))\left\{\begin{array}{l}
\subseteq \operatorname{cores} \mathcal{P}(\widetilde{\operatorname{Mod} R}), \\
\subseteq{ }^{\perp}(\mathcal{P}(\operatorname{Mod} R)) \supseteq{ }^{\perp}(\mathcal{F}(\operatorname{Mod} R)) \supseteq \mathcal{S G \mathcal { F }}(\operatorname{Mod} R)
\end{array}\right.
$$

and

$\mathcal{I}(\operatorname{Mod} R) \subseteq \mathcal{G} \mathcal{F} \mathcal{I}(\operatorname{Mod} R)$

$$
\subseteq \mathcal{G}(\mathcal{I}(\operatorname{Mod} R))\left\{\begin{array}{l}
\subseteq \operatorname{res} \mathcal{I}(\widetilde{\operatorname{Mod} R}) \\
\subseteq(\mathcal{I}(\operatorname{Mod} R))^{\perp} \supseteq(\mathcal{F} \mathcal{I}(\operatorname{Mod} R))^{\perp} \supseteq \mathcal{G F \mathcal { I }}(\operatorname{Mod} R)
\end{array}\right.
$$

So for any module $A$ in $\operatorname{Mod} R$, we have that

$$
\begin{aligned}
\operatorname{pd}_{R} A & \geq \operatorname{SGfd}_{R} A \\
& \geq \operatorname{Gpd}_{R} A\left\{\begin{array}{l}
\geq \operatorname{cores} \mathcal{P}(\widetilde{\operatorname{Mod} R})-\operatorname{dim} A \\
\geq \perp(\mathcal{P}(\operatorname{Mod} R))-\operatorname{dim} A \leq{ }^{\perp}(\mathcal{F}(\operatorname{Mod} R))-\operatorname{dim} A \leq \operatorname{SGfd}_{R} A
\end{array}\right.
\end{aligned}
$$


and

$\operatorname{id}_{R} A \geq \operatorname{GFid}_{R} A$

$\geq \operatorname{Gid}_{R} A\left\{\begin{array}{l}\geq \operatorname{res} \widetilde{\mathcal{I}(\widehat{\operatorname{Mod} R})-\operatorname{codim} A} \\ \geq(\mathcal{I}(\operatorname{Mod} R))^{\perp}-\operatorname{codim} A \leq(\mathcal{F I}(\operatorname{Mod} R))^{\perp}-\operatorname{codim} A \leq \operatorname{GFid}_{R} A .\end{array}\right.$

\section{THEOREM 5.6}

Let $A$ be a module in $\operatorname{Mod} R$.

(a) If $A \in(\mathcal{S G F}(\operatorname{Mod} R))^{\perp}$, then $\operatorname{pd}_{R} A=\operatorname{SGfd}_{R} A$.

(b) If $A \in(\mathcal{G}(\mathcal{P}(\operatorname{Mod} R)))^{\perp}$, then $\operatorname{pd}_{R} A=\operatorname{SGfd}_{R} A=\operatorname{Gpd}_{R} A$. $\operatorname{dim} A$.

(c) If $\operatorname{id}_{R} A<\infty$, then $\operatorname{pd}_{R} A=\operatorname{SGfd}_{R} A=\operatorname{Gpd}_{R} A=\operatorname{cores} \mathcal{P} \widetilde{(\operatorname{Mod} R)}$ -

(d) If $\operatorname{pd}_{R} A<\infty$, then $\operatorname{pd}_{R} A=\operatorname{SGfd}_{R} A=\operatorname{Gpd}_{R} A={ }^{\perp}(\mathcal{P}(\operatorname{Mod} R))$ $\operatorname{dim} A={ }^{\perp}(\mathcal{F}(\operatorname{Mod} R))-\operatorname{dim} A$.

(e) If $\operatorname{SGfd}_{R} A<\infty$, then $\operatorname{SGfd}_{R} A=\operatorname{Gpd}_{R} A={ }^{\perp}(\mathcal{P}(\operatorname{Mod} R))-\operatorname{dim} A=$ $\perp(\mathcal{F}(\operatorname{Mod} R))-\operatorname{dim} A$.

(f) If $\operatorname{Gpd}_{R} A<\infty$, then $\operatorname{Gpd}_{R} A={ }^{\perp}(\mathcal{P}(\operatorname{Mod} R))-\operatorname{dim} A$.

(g) If $\mathrm{fd}_{R} A<\infty$, then $\operatorname{pd}_{R} A=\operatorname{SGfd}_{R} A$.

\section{Proof}

(a) (resp., (b)) It is clear that $\mathcal{P}(\operatorname{Mod} R)$ is both a $\mathcal{P}(\operatorname{Mod} R)$-proper generator and a $\mathcal{P}(\operatorname{Mod} R)$-coproper cogenerator for $\mathcal{S G \mathcal { F }}(\operatorname{Mod} R)$ (resp., $\mathcal{G}(\mathcal{P}(\operatorname{Mod} R))$ ). Note that $\mathcal{S G \mathcal { F }}(\operatorname{Mod} R)(\operatorname{resp} ., \mathcal{G}(\mathcal{P}(\operatorname{Mod} R)))$ is closed under extensions by $[\mathrm{Hu}$, Lemma 3.1] (resp., [H1, Theorem 2.5]). Then by putting $\mathscr{T}=\mathcal{S G \mathcal { F }}(\operatorname{Mod} R)$ (resp., $\mathscr{T}=\mathcal{G}(\mathcal{P}(\operatorname{Mod} R))$ ) and $\mathscr{C}=\mathcal{P}(\operatorname{Mod} R)$ in Theorem 3.10(c), we have $\operatorname{pd}_{R} A=\operatorname{SGfd}_{R} A\left(\right.$ resp., $\left.\operatorname{pd}_{R} A=\operatorname{Gpd}_{R} A\right)$ if $A \in(\mathcal{S G \mathcal { F }}(\operatorname{Mod} R))^{\perp}$ (resp., $A \in$ $\left.(\mathcal{G}(\mathcal{P}(\operatorname{Mod} R)))^{\perp}\right)$.

(c) It is clear that $\mathcal{P}(\operatorname{Mod} R)$ is a $\mathcal{P}(\operatorname{Mod} R)$-coproper cogenerator for cores $\mathcal{P}(\widetilde{\operatorname{Mod}} R)$. Note that cores $\mathcal{P}(\widetilde{\operatorname{Mod}} R)$ is closed under $\mathcal{P}(\operatorname{Mod} R)$-coproper extensions by $[\mathrm{Hu}$, Lemma 3.1]. Then by putting $\mathscr{T}=\operatorname{cores} \mathcal{P}(\widehat{\operatorname{Mod} R})$, $\mathscr{C}=\mathcal{P}(\operatorname{Mod} R)$, and $\mathscr{D}=\mathcal{I}(\operatorname{Mod} R)$ in Theorem 4.11, we have $\operatorname{pd}_{R} A=$ cores $\mathcal{P}(\widehat{\operatorname{Mod}} R)-\operatorname{dim} A$ if $\operatorname{id}_{R} A<\infty$.

(d) By Proposition 5.3(a), we have $\operatorname{pd}_{R} A={ }^{\perp}(\mathcal{P}(\operatorname{Mod} R))-\operatorname{dim} A$ if $\operatorname{pd}_{R} A<\infty$.

(e) Note that $\mathcal{S G \mathcal { F }}(\operatorname{Mod} R)$ is closed under extensions (see the proof of (a)). Let $\operatorname{SGfd}_{R} A=n<\infty$. Then by Theorem 3.6, there exists an exact sequence

$$
0 \rightarrow G_{n} \rightarrow P_{n-1} \rightarrow P_{n-2} \rightarrow \cdots \rightarrow P_{0} \rightarrow A \rightarrow 0
$$

in $\operatorname{Mod} R$ with all $P_{i}$ 's projective and $G_{n}$ strongly Gorenstein flat. Put $K_{i}=$ $\operatorname{Im}\left(P_{i} \rightarrow P_{i-1}\right)$ for any $1 \leq i \leq n-1$. Suppose that ${ }^{\perp}(\mathcal{P}(\operatorname{Mod} R))-\operatorname{dim} A=m<$ $\infty$. It suffices to show that $m \geq n$. If $m<n$, then $K_{m} \in{ }^{\perp}(\mathcal{P}(\operatorname{Mod} R))$ and $K_{n-1} \in$ 
${ }^{\perp}(\mathcal{P}(\operatorname{Mod} R))$ by Theorem 3.8(a). Because there exists a $\operatorname{Hom}_{R}(-, \mathcal{F}(\operatorname{Mod} R))$ exact exact sequence $0 \rightarrow G_{n} \rightarrow P \rightarrow G \rightarrow 0$ in $\operatorname{Mod} R$ with $P$ projective and $G$ strongly Gorenstein flat, we have the following pushout diagram:

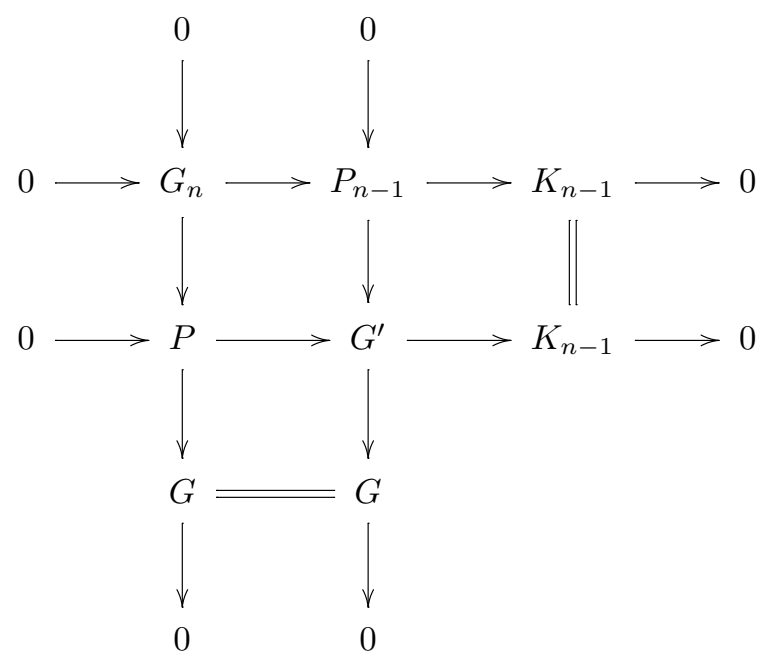

By using an argument similar to that in the proof of [H1, Theorem 2.5], we get that $\mathcal{S G \mathcal { F }}(\operatorname{Mod} R)$ is closed under direct summands. Because both the middle column and the middle row in the above diagram split, $G^{\prime} \cong P_{n-1} \oplus G$ is strongly Gorenstein flat and $K_{n-1}$ is isomorphic to a direct summand of $G^{\prime}$, which implies that $K_{n-1}$ is strongly Gorenstein flat and $\operatorname{SGfd}_{R} A \leq n-1$. It is a contradiction.

(f) We get the assertion by putting $\mathscr{C}=\mathcal{P}(\operatorname{Mod} R)$ in Theorem 3.14 or $\mathscr{C}=$ $\mathcal{P}(\operatorname{Mod} R)$ and $\mathscr{T}=\mathcal{G}(\mathcal{P}(\operatorname{Mod} R))$ in Theorem 3.10.

(g) By the definition of strongly Gorenstein flat modules, it is easy to see that $A \in(\mathcal{S G F}(\operatorname{Mod} R))^{\perp}$ if $\mathrm{fd}_{R} A<\infty$. Then the assertion follows from (a).

\section{REMARK 5.7}

Theorem 5.6(b) is [Z, Theorem 2.3]. Theorem 5.6(c) generalizes [H2, Theorem 2.2], which states that for a module $A$ in $\operatorname{Mod} R$, if id $\operatorname{id}_{R} A<\infty$, then $\operatorname{pd}_{R} A=$ $\operatorname{Gpd}_{R} A$. Notice that a module in $\operatorname{Mod} R$ with finite injective dimension is in $(\mathcal{G}(\mathcal{P}(\operatorname{Mod} R)))^{\perp}$, so we may also get [H2, Theorem 2.2] by Theorem 5.6(b) (see [Z, Corollary 2.5]). Theorem 5.6(f) is well known (see [H1, Theorem 2.20]).

Let $A$ be a module in $\operatorname{Mod} R$. Recall that $A$ is called Gorenstein flat if there exists an exact sequence

$$
\cdots \rightarrow F_{1} \rightarrow F_{0} \rightarrow F^{0} \rightarrow F^{1} \rightarrow \cdots
$$

in $\operatorname{Mod} R$ with all terms flat such that $A \cong \operatorname{Im}\left(F_{0} \rightarrow F^{0}\right)$ and the sequence remains still exact after applying the functor $I \otimes_{R}-$ for any injective right $R$ module $I$. The Gorenstein flat dimension of $A$, denoted by $\operatorname{Gfd}_{R} A$, is defined to be $\inf \left\{n \mid\right.$ there exists an exact sequence $0 \rightarrow H_{n} \rightarrow \cdots \rightarrow H_{1} \rightarrow H_{0} \rightarrow A \rightarrow 0$ with all $H_{i}$ 's Gorenstein flat\}. Set $\operatorname{Gfd}_{R} A=\infty$ if no such $n$ exists (see [EJT], [H1]). 
The following is an open question: is every Gorenstein projective module over any ring Gorenstein flat? Holm proved in [H1, Proposition 2.4] that if $R$ is a right coherent ring with finite left finitistic projective dimension, then every Gorenstein projective module in $\operatorname{Mod} R$ is Gorenstein flat. As an immediate consequence of Theorem 5.6, we have the following.

\section{COROLLARY 5.8}

Let $R$ be a right coherent ring, and let $A$ be a module in $\operatorname{Mod} R$. Then $\operatorname{Gpd}_{R} A \geq$ $\operatorname{Gfd}_{R} A$ if either of the following conditions is satisfied:

(a) $A \in(\mathcal{G}(\mathcal{P}(\operatorname{Mod} R)))^{\perp}$ (in particular, if $\operatorname{pd}_{R} A<\infty$ or $\left.\operatorname{id}_{R} A<\infty\right)$, or

(b) $\operatorname{SGfd}_{R} A<\infty$.

\section{Proof}

Let $R$ be a right coherent ring, and let $A$ be a module in $\operatorname{Mod} R$. Then $\operatorname{SGfd}_{R} A \geq$ $\operatorname{Gfd}_{R} A$ by [DLM, Proposition 2.3]. So the assertions follow from Theorems 5.6(b) and 5.6(e), respectively.

Recall from [B1] that $R$ is called left GF-closed if the subcategory of $\operatorname{Mod} R$ consisting of Gorenstein flat modules is closed under extensions.

\section{COROLLARY 5.9}

Let $A$ be a module in $\operatorname{Mod} R$, and let $n$ be a nonnegative integer.

(a) ([CFH, Lemma 2.17]) If $\operatorname{Gpd}_{R} A=n$, then there exists an exact sequence $0 \rightarrow A \rightarrow B \rightarrow T \rightarrow 0$ in $\operatorname{Mod} R$ with $\operatorname{pd}_{B} A=n$ and $T$ Gorenstein projective.

(b) If $\operatorname{SGfd}_{R} A=n$, then there exists an exact sequence $0 \rightarrow A \rightarrow B \rightarrow T \rightarrow 0$ in $\operatorname{Mod} R$ with $\operatorname{pd}_{B} A=n$ and $T$ strongly Gorenstein flat.

(c) If $R$ is left $G F$-closed and $\operatorname{Gfd}_{R} A=n$, then there exists an exact sequence $0 \rightarrow A \rightarrow B \rightarrow T \rightarrow 0$ in $\operatorname{Mod} R$ with $\operatorname{fd}_{R} B=n$ and $T$ Gorenstein flat.

\section{Proof}

(a) (resp., (b)) Let $\operatorname{Gpd}_{R} A=n$ (resp., $\operatorname{SGfd}_{R} A=n$ ). By Corollary 4.5, there exists an exact sequence $0 \rightarrow A \rightarrow B \rightarrow T \rightarrow 0$ in $\operatorname{Mod} R$ with $\operatorname{pd}_{R} B \leq n$ and $T$ Gorenstein projective (resp., strongly Gorenstein flat). Then by [H1, Theorem 2.5] (resp., Example 3.2(d)) and Proposition 2.3, we have $\operatorname{Gpd}_{R} B \geq$ $\operatorname{Gpd}_{R} A=n\left(\right.$ resp., $\left.\operatorname{SGfd}_{R} B \geq \operatorname{SGfd}_{R} A=n\right)$. So $\operatorname{pd}_{R} B=n$ by Theorem $5.6(\mathrm{~d})$.

(c) Let $R$ be left GF-closed, and let $\operatorname{Gfd}_{R} A=n$. By Corollary 4.5, there exists an exact sequence $0 \rightarrow A \rightarrow B \rightarrow T \rightarrow 0$ in $\operatorname{Mod} R$ with $\operatorname{fd}_{R} B \leq n$ and $T$ Gorenstein flat. Then by [B1, Theorem 2.3] and Proposition 2.3, we have that $\operatorname{Gfd}_{R} B \geq \operatorname{Gfd}_{R} A=n$. So $\operatorname{fd}_{R} B=n$ by [B2, Theorem 2.2].

Recall that the FP-injective dimension $\mathrm{FP}^{-i d}{ }_{R} A$ of $A$ in $\operatorname{Mod} R$ is defined to be $\inf \left\{n \mid\right.$ there exists an exact sequence $0 \rightarrow A \rightarrow E^{0} \rightarrow E^{1} \rightarrow \cdots \rightarrow E^{n} \rightarrow 0$ in $\operatorname{Mod} R$ with all $E^{i}$ in $\left.\mathcal{F} \mathcal{I}(\operatorname{Mod} R)\right\}$. Set $\mathrm{FP}-i_{R} A=\infty$ if no such $n$ exists. 
The following result is the dual of Theorem 5.6.

\section{THEOREM 5.10}

Let $A$ be a module in $\operatorname{Mod} R$.

(a) If $A \in{ }^{\perp}(\mathcal{G} \mathcal{F} \mathcal{I}(\operatorname{Mod} R))$, then $\operatorname{id}_{R} A=\operatorname{GFid}_{R} A$.

(b) If $A \in{ }^{\perp}(\mathcal{G}(\mathcal{I}(\operatorname{Mod} R)))$, then $\operatorname{id}_{R} A=\operatorname{GFid}_{R} A=\operatorname{Gid}_{R} A$.

(c) If $\operatorname{pd}_{R} A<\infty$, then $\operatorname{id}_{R} A=\operatorname{GFid}_{R} A=\operatorname{Gid}_{R} A=\operatorname{res} \widetilde{\mathcal{I}(\widetilde{\operatorname{Mod}} R)}$ $\operatorname{codim} A$.

(d) If $\operatorname{id}_{R} A<\infty$, then $\operatorname{id}_{R} A=\operatorname{GFid}_{R} A=\operatorname{Gid}_{R} A=(\mathcal{I}(\operatorname{Mod} R))^{\perp}$ $\operatorname{codim} A=(\mathcal{F} \mathcal{I}(\operatorname{Mod} R))^{\perp}-\operatorname{codim} A$.

(e) If $\operatorname{GFid}_{R} A<\infty$, then $\operatorname{GFid}_{R} A=\operatorname{Gid}_{R} A=(\mathcal{I}(\operatorname{Mod} R))^{\perp}-\operatorname{codim} A=$ $(\mathcal{F} \mathcal{I}(\operatorname{Mod} R))^{\perp}-\operatorname{codim} A$.

(f) If $\operatorname{Gid}_{R} A<\infty$, then $\operatorname{Gid}_{R} A=(\mathcal{I}(\operatorname{Mod} R))^{\perp}-\operatorname{codim} A$.

(g) If FP-id ${ }_{R} A<\infty$, then $\operatorname{id}_{R} A=\operatorname{GFid}_{R} A$.

REMARK 5.11

Theorem 5.10(c) generalizes [H2, Theorem 2.1], which states that for a module $A$ in $\operatorname{Mod} R$, if $\operatorname{pd}_{R} A<\infty$, then $\operatorname{id}_{R} A=\operatorname{Gid}_{R} A$. Notice that a module in $\operatorname{Mod} R$ with finite projective dimension is in ${ }^{\perp}(\mathcal{G}(\mathcal{I}(\operatorname{Mod} R)))$, so we may also get [H2, Theorem 2.1] by Theorem 5.10(b). Theorem 5.10(f) is well known (see [H1, Theorem 2.22]).

\subsection{Questions}

In view of Theorems 5.6(c) and 5.6(d), it is natural to ask the following.

QUESTION 5.12

If $A$ is a module in $\operatorname{Mod} R$ with $\operatorname{id}_{R} A<\infty, \operatorname{does~}_{\operatorname{pd}_{R}} A=^{\perp}(\mathcal{P}(\operatorname{Mod} R))-\operatorname{dim} A$ hold?

\section{QUESTION 5.13}

If $A$ is a module in $\operatorname{Mod} R$ with $\operatorname{pd}_{R} A<\infty, \operatorname{does} \operatorname{pd}_{R} A=\operatorname{cores} \mathcal{P}(\widetilde{\operatorname{Mod}} R)-\operatorname{dim} A$ hold?

From now on, $R$ is a left and right Noetherian ring (unless stated otherwise). We write ${ }^{\perp} R=\left\{M \in \bmod R \mid \operatorname{Ext}_{R}^{i}\left({ }_{R} M,{ }_{R} R\right)=0\right.$ for any $\left.i \geq 1\right\}$ (resp., ${ }^{\perp} R_{R}=$ $\left\{N \in \bmod R^{\text {op }} \mid \operatorname{Ext}_{R^{\text {op }}}^{i}\left(N_{R}, R_{R}\right)=0\right.$ for any $\left.\left.i \geq 1\right\}\right)$.

For any module $A$ in $\bmod R$, there exists a projective presentation

$$
P_{1} \stackrel{f}{\longrightarrow} P_{0} \rightarrow A \rightarrow 0
$$

of $A$ in $\bmod R$. (Note that if $R$ is an Artinian algebra, then this projective presentation of $A$ is chosen to be the minimal one.) Then we get an exact sequence

$$
0 \rightarrow A^{*} \rightarrow P_{0}^{*} \stackrel{f^{*}}{\rightarrow} P_{1}^{*} \rightarrow \operatorname{Tr} A \rightarrow 0
$$


in $\bmod R^{\mathrm{op}}$, where $(-)^{*}=\operatorname{Hom}(-, R)$ and $\operatorname{Tr} A=$ Coker $f^{*}$ is the transpose of $A$. Auslander and Bridger generalized the notions of finitely generated projective modules and the projective dimension of finitely generated modules as follows. A module $A$ in $\bmod R$ is said to have Gorenstein dimension zero if $A \in{ }^{\perp} R$ and $\operatorname{Tr} A \in{ }^{\perp} R_{R}$ (see [AB]). It is well known that, over a left and right Noetherian ring, a finitely generated module is Gorenstein projective if and only if it has Gorenstein dimension zero (see [EJ2, Proposition 10.2.6]).

Let $A$ be a module in $\bmod R$. Recall from [HuH] that $A$ is called $\infty$-torsionfree if $\operatorname{Tr} A \in{ }^{\perp} R_{R}$. We use $\mathcal{T}(\bmod R)$ to denote the subcategory of $\bmod R$ consisting of $\infty$-torsion-free modules. The torsion-free dimension of $A$, denoted by $\mathcal{T}-\operatorname{dim}_{R} A$, is defined to be $\inf \left\{n \mid\right.$ there exists an exact sequence $0 \rightarrow X_{n} \rightarrow \cdots \rightarrow$ $X_{1} \rightarrow X_{0} \rightarrow A \rightarrow 0$ in $\bmod R$ with all $X_{i}$ 's in $\left.\mathcal{T}(\bmod R)\right\}$. Set $\mathcal{T}-\operatorname{dim}_{R} A=\infty$ if no such $n$ exists. By [AB, Theorem 2.17], a module is in cores $\widehat{\mathcal{P}(\bmod R)}$ if and only if it is in $\mathcal{T}(\bmod R)$. So cores $\mathcal{P}(\widehat{\bmod R} R)-\operatorname{dim} A=\mathcal{T}-\operatorname{dim}_{R} A$ for any module $A$ in $\bmod R$.

By Example 4.2(e) and Corollary 4.5, we immediately have the following.

\section{COROLLARY 5.14 ([HuH, COROLLARY 3.5])}

Let $A$ be a module in $\bmod R$ with $\mathcal{T}-\operatorname{dim}_{R} A=n$. Then there exists an exact sequence $0 \rightarrow A \rightarrow B \rightarrow T \rightarrow 0$ in $\bmod R$ with $\operatorname{pd}_{R} B \leq n$ and $T \infty$-torsion-free.

The following result is analogous to Theorems 5.6(c) and 5.6(d).

\section{THEOREM 5.15}

Let $R$ be a left and right Noetherian ring, and let $A$ be a module in $\bmod R$.

(a) If $\operatorname{id}_{R} A<\infty$, then $\operatorname{pd}_{R} A=\operatorname{Gpd}_{R} A=\mathcal{T}-\operatorname{dim}_{R} A$.

(b) If $\operatorname{pd}_{R} A<\infty$, then $\operatorname{pd}_{R} A=\operatorname{Gpd}_{R} A={ }^{\perp} R$ - $\operatorname{dim} A$.

In view of the assertions in Theorem 5.15, it is natural to ask the following questions, which are finitely generated versions of Questions 5.12 and 5.13, respectively.

\section{QUESTION 5.16}

If $A$ is a module in $\bmod R$ with $\operatorname{id}_{R} A<\infty$, does $\operatorname{pd}_{R} A={ }^{\perp} R$ - $\operatorname{dim} A$ hold? (Equivalently, does $\operatorname{Gpd}_{R} A={ }^{\perp} R$ - $\operatorname{dim} A$ hold?)

\section{QUESTION 5.17}

If $A$ is a module in $\bmod R$ with $\operatorname{pd}_{R} A<\infty$, does $\operatorname{pd}_{R} A=\mathcal{T}$ - $\operatorname{dim}_{R} A$ hold? (Equivalently, does $\operatorname{Gpd}_{R} A=\mathcal{T}$ - $\operatorname{dim}_{R} A$ hold?)

Let $R$ be an Artinian algebra, let $C(R)$ be the center of $R$, let $J$ be the Jacobson radical of $C(R)$, and let $I(C(R) / J)$ be the injective envelope of $C(R) / J$. Then the Matlis duality $\mathbb{D}(-)=\operatorname{Hom}_{C(R)}(-, I(C(R) / J))$ between $\bmod R$ and 
$\bmod R^{\mathrm{op}}$ induces a duality between projective (resp., injective) modules in $\bmod R$ and injective (resp., projective) modules in $\bmod R^{\text {op }}$. As a special case of Question 5.16, we propose the following.

\section{CONJECTURE 5.18}

Let $R$ be an Artinian algebra.

(a) A module $A$ in $\bmod R$ is projective if $A$ is injective and $A \in{ }_{R}^{\perp} R$.

(b) $R$ is self-injective if $\mathbb{D}\left(R_{R}\right) \in{ }^{\perp}{ }_{R} R$.

The Auslander-Reiten conjecture (ARC for short) states that, over any Artinian algebra $R$, a module $A$ in $\bmod R$ is projective if $\operatorname{Ext}_{R}^{i}(A \oplus R, A \oplus R)=0$ for any $i \geq 1$ (see $[\mathrm{AR}]$ ). The strong Nakayama conjecture (SNC for short) states that, over any Artinian algebra $R$, for any $0 \neq A$ in $\bmod R$ there exists an $i \geq 0$ such that $\operatorname{Ext}_{R}^{i}(A, R) \neq 0$ (see $[\mathrm{CoF}]$ ). These two conjectures still remain open. Observe that an equivalent version of ARC states that, over any Artinian algebra $R$, for any simple module $S$ in $\bmod R$ there exists $i \geq 0$ such that $\operatorname{Ext}_{R}^{i}(S, R) \neq 0$ (see $[\mathrm{AR}]$ ). So $\mathrm{SNC} \Rightarrow \mathrm{ARC}$. It is easy to see that $\mathrm{ARC} \Rightarrow \mathrm{Con}-$ jecture $5.18(\mathrm{a}) \Rightarrow$ Conjecture $5.18(\mathrm{~b})$.

The following result shows that Question 5.17 is closely related to SNC.

PROPOSITION 5.19

Let $R$ be an Artinian algebra. Then the following statements are equivalent.

(a) SNC holds for $R^{\mathrm{op}}$.

(b) $A$ module in $\bmod R$ is projective if $A \in \mathcal{T}(\bmod R)$ and $\operatorname{pd}_{R} A \leq 1$.

Proof

(a) $\Rightarrow$ (b) Let $A \in \mathcal{T}(\bmod R)$, and let $\operatorname{pd}_{R} A \leq 1$. Then $\operatorname{Tr} A \in{ }^{\perp} R_{R}$ and there exists a minimal projective presentation

$$
0 \rightarrow P_{1} \rightarrow P_{0} \rightarrow A \rightarrow 0
$$

in $\bmod R$, which induces an exact sequence

$$
0 \rightarrow A^{*} \rightarrow P_{0}^{*} \rightarrow P_{1}^{*} \rightarrow \operatorname{Tr} A \rightarrow 0
$$

in $\bmod R^{\mathrm{op}}$. So we get the following commutative diagram with exact rows:

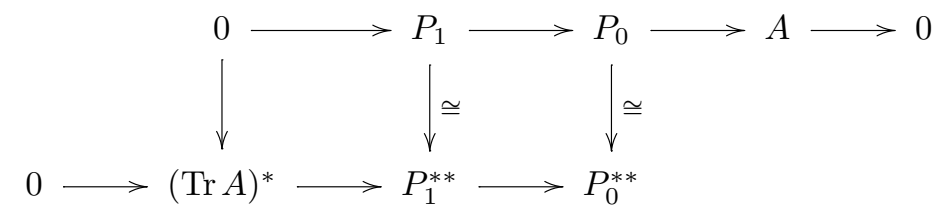

Thus $(\operatorname{Tr} A)^{*}=0$ and $\operatorname{Ext}_{R}^{i}(\operatorname{Tr} A, R)=0$ for any $i \geq 0$. Then $\operatorname{Tr} A=0$ by (a), which implies that $A$ is projective by [ARS, Chapter IV, Proposition 1.7(b)].

(b) $\Rightarrow$ (a) Let $B$ be a module in $\bmod R^{\text {op }}$ such that $\operatorname{Ext}_{R^{\text {op }}}^{i}(B, R)=0$ for any $i \geq 0$. Then $B$ has no nonzero projective summands. So $B \cong \operatorname{Tr} \operatorname{Tr} B$ by 
[ARS, Chapter IV, Proposition 1.7(c)] and hence $\operatorname{Ext}_{R^{\text {op }}}^{i}(\operatorname{Tr} \operatorname{Tr} B, R)=0$ for any $i \geq 0$. So $\operatorname{Tr} B \in \mathcal{T}(\bmod R)$. From a minimal projective presentation $Q_{1} \rightarrow Q_{0} \rightarrow$ $B \rightarrow 0$ of $B$ in $\bmod R^{\text {op }}$, we get an exact sequence

$$
0 \rightarrow B^{*} \rightarrow Q_{0}^{*} \rightarrow Q_{1}^{*} \rightarrow \operatorname{Tr} B \rightarrow 0
$$

in $\bmod R$ with $Q_{0}^{*}, Q_{1}^{*}$ projective. Because $B^{*}=0, \operatorname{pd}_{R} \operatorname{Tr} B \leq 1$. Then $\operatorname{Tr} B$ is projective by (b), which implies that $B$ is projective. Again because $B^{*}=0$, $B=0$. Therefore SNC holds for $R^{\text {op }}$.

Acknowledgment. The author thanks the referee for the useful suggestions.

\section{References}

[AB] M. Auslander and M. Bridger, Stable Module Theory, Mem. Amer. Math. Soc. 94, Amer. Math. Soc., Providence, 1969. MR 0269685.

[AR] M. Auslander and I. Reiten, On a generalized version of the Nakayama conjecture, Proc. Amer. Math. Soc. 52 (1975), 69-74. MR 0389977.

[ARS] M. Auslander, I. Reiten, and S. O. Smolø, Representation Theory of Artin Algebras, corrected reprint, Cambridge Stud. Adv. Math. 36, Cambridge Univ. Press, Cambridge, 1997. MR 1476671.

[B1] D. Bennis, Rings over which the class of Gorenstein flat modules is closed under extensions, Comm. Algebra 37 (2009), 855-868. MR 2503181. DOI 10.1080/00927870802271862.

[B2] _ A note on Gorenstein flat dimension, Algebra Colloq. 18 (2011), 155-161. MR 2753665. DOI 10.1142/S1005386711000095.

[C] L. W. Christensen, Gorenstein Dimensions, Lecture Notes in Math. 1747, Springer, Berlin, 2000. MR 1799866. DOI 10.1007/BFb0103980.

[CFH] L. W. Christensen, A. Frankild, and H. Holm, On Gorenstein projective, injective and flat dimensions - a functorial description with applications, J. Algebra 302 (2006), 231-279. MR 2236602.

DOI 10.1016/j.jalgebra.2005.12.007.

[CI] L. W. Christensen and S. Iyengar, Gorenstein dimension of modules over homomorphisms, J. Pure Appl. Algebra 208 (2007), 177-188. MR 2269838. DOI 10.1016/j.jpaa.2005.12.005.

[CoF] R. R. Colby and K. R. Fuller, A note on the Nakayama conjectures, Tsukuba J. Math. 14 (1990), 343-352. MR 1085203.

[DLM] N. Ding, Y. Li, and L. Mao, Strongly Gorenstein flat modules, J. Aust. Math. Soc. 86 (2009), 323-338. MR 2529328. DOI 10.1017/S1446788708000761.

[EJ1] E. E. Enochs and O. M. G. Jenda, Gorenstein injective and projective modules, Math. Z. 220 (1995), 611-633. MR 1363858. DOI 10.1007/BF02572634.

[EJ2] - Relative Homological Algebra, de Gruyter Exp. Math. 30, de Gruyter, Berlin, 2000. MR 1753146. DOI 10.1515/9783110803662. 
[EJL] E. E. Enochs, O. M. G. Jenda, and J. A. López-Ramos, Covers and envelopes by $V$-Gorenstein modules, Comm. Algebra 33 (2005), 4705-4717.

MR 2188336. DOI 10.1080/00927870500328766.

[EJT] E. E. Enochs, O. M. G. Jenda, and B. Torrecillas, Gorenstein flat modules, Nanjing Daxue Xuebao Shuxue Bannian Kan 10 (1993), 1-9. MR 1248299.

[GD] Y. Geng and N. Ding, $\mathcal{W}$-Gorenstein modules, J. Algebra 325 (2011), 132-146. MR 2745532. DOI 10.1016/j.jalgebra.2010.09.040.

[GT] R. Göbel and J. Triifaj, Approximations and Endomorphism Algebras of Modules, de Gruyter Exp. Math. 41, de Gruyter, Berlin, 2006. MR 2251271.

[H1] H. Holm, Gorenstein homological dimensions, J. Pure Appl. Algebra 189 (2004), 167-193. MR 2038564. DOI 10.1016/j.jpaa.2003.11.007.

[H2] Rings with finite Gorenstein injective dimension, Proc. Amer. Math. Soc. 132 (2004), 1279-1283. MR 2053331.

DOI 10.1090/S0002-9939-03-07466-5.

[Hu] Z. Huang, Proper resolutions and Gorenstein categories, J. Algebra 393 (2013), 142-169. MR 3090064. DOI 10.1016/j.jalgebra.2013.07.008.

$[\mathrm{HuH}] \quad$ C. Huang and Z. Huang, Torsionfree dimension of modules and self-injective dimension of rings, Osaka J. Math. 49 (2012), 21-35. MR 2903252.

[LHX] Z. Liu, Z. Huang, and A. Xu, Gorenstein projective dimension relative to a semidualizing bimodule, Comm. Algebra 41 (2013), 1-18. MR 3010518. DOI 10.1080/00927872.2011.602782.

[MD] L. Mao and N. Ding, Gorenstein FP-injective and Gorenstein flat modules, J. Algebra Appl. 7 (2008), 491-506. MR 2442073.

DOI 10.1142/S0219498808002953.

[SSW] S. Sather-Wagstaff, T. Sharif, and D. White, Stability of Gorenstein categories, J. Lond. Math. Soc. (2) 77 (2008), 481-502. MR 2400403. DOI $10.1112 / \mathrm{jlms} / \mathrm{jdm} 124$.

[Z] P. Zhang, Gorensteinness and Buchweitz theorem, preprint, 2012.

Department of Mathematics, Nanjing University, Nanjing 210093, Jiangsu Province, China; huangzy@nju.edu.cn 